\title{
Existence and continuity of optimal solutions to some structural topology optimization problems including unilateral constraints and stochastic loads*
}

\author{
Michael Patriksson ${ }^{\dagger}$ and Joakim Petersson ${ }^{\ddagger}$
}

September 26, 2001

\begin{abstract}
We consider a general discrete structural optimization problem including unilateral constraints arising from, for example, non-penetration conditions in contact mechanics or noncompression conditions for elastic ropes. The loads applied (and, in principle, also other data such as the initial distances to the supports), are allowed to be stochastic, which we handle through a discretization of the probability space. The existence of optimal solutions to the resulting problem is established, as well as the continuity properties of the equilibrium displacements and forces with respect to the lower bounds on the design variables. The latter feature is important in topology optimization, in which one includes the possibility of vanishing structural parts by setting design variable values to zero. In design optimization computations, one usually replaces the zero lower design bound by a strictly positive number, hence rewriting the problem into a sizing form. For several such perturbations, we prove that the global optimal designs and equilibrium states converge to the correct ones as the lower bound converges to zero.
\end{abstract}

\section{Introduction}

\subsection{Motivation}

Topology optimization of mechanical structures refers to the subfield of structural optimization where parts of the design region are allowed to be occupied by a varying amount of solid material, including no material at all. This means that the sets of admissible designs and the corresponding structural responses are very large. On the one hand, some designs might result in a structure that cannot carry the applied load at all, while, on the other hand, some designs carry the particular load very efficiently. The distinction between sizing and topology optimization is usually that in the latter the amount of material, for example, a thickness or cross-sectional area, is allowed to be zero. Figure 1 shows a simple one-dimensional structure ${ }^{1}$ that consists of a bar suspended with one cable. Suppose that one has chosen the bar material volume (or the cross-sectional area) $x$ and the cable material volume $X$ to be design variables. If the objective is to maximize the displacement

*This research is supported by a grant from the Swedish Research Council for Engineering Sciences (grant TFR 98-125), which is greatfully acknowledged.

${ }^{\dagger}$ Department of Mathematics, Chalmers University of Technology, SE-412 96 Göteborg, Sweden. Email: mipat@math.chalmers.se

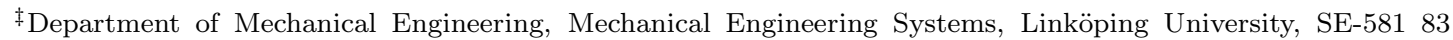
Linköping, Sweden. Email: joape@ikp.liu.se

${ }^{1}$ This example will be studied in more detail in Section 3.1.2. 


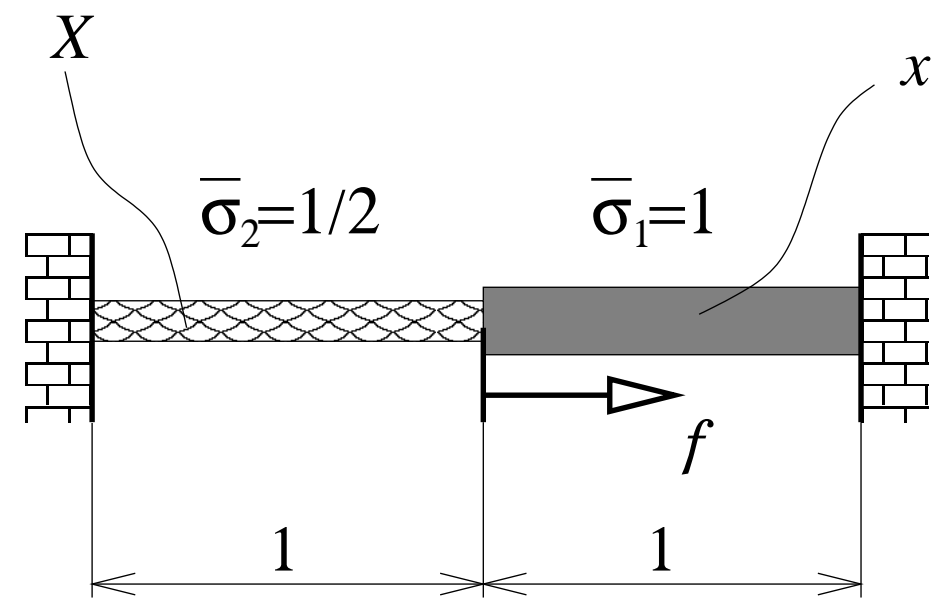

Figure 1: The cable suspended one-bar truss.

in the middle, then there are in fact no optimal solutions in the topology optimization problem unlike in the corresponding sizing optimization problem. The reason is that the objective value can be made arbitrarily large by choosing $x$ and $X$ arbitrarily close to zero (which is not allowed in the sizing case). Although the objective function is somewhat artificial, similar phenomena can occur also in a more natural context, and it illustrates that it is in general not obvious that there exist solutions to topology optimization problems as some mathematical properties are different from the sizing case. In this example, the key issue can be described by a lack of closedness of the feasible set (in the problem statement involving both the state and design variables), which has been observed earlier to be an important factor in establishing existence of solutions in general mathematical programs with equilibrium constraints in, e.g., [LPR96, Section 1.4].

Sizing problems are typically easier than topology optimization problems for more reasons than that the existence issue is easier - for instance the design sensitivities, that is, the derivatives of the state variables (in the nested version of the problem) with respect to changes in design, are harder to determine in other problem types such as in shape optimization. Therefore, if topology optimization problems could be cast into a sizing-like problem statement, then the problem would be much easier to handle, since design sensitivities and unique equilibrium solutions always exist and are computable. The traditional way to restate or modify the problem statement from a topology optimization problem to a sizing-like statement is here referred to as an $\varepsilon$-perturbation (or, an $\varepsilon$-relaxation). In order for the $\varepsilon$-perturbation to be valid, the solution sets of the restated sizing-like problems should be close to those of the original problem statement for small parameter values $\varepsilon$. Difficulties in finding and validating proper $\varepsilon$-perturbations should somehow be expected since the unperturbed problem statements include many different structural topologies whereas the sizing problems cover only one.

The most common $\varepsilon$-perturbation is to replace the design zero lower bounds by a small positive number $\varepsilon$ - a perturbation which is valid for some minimum compliance problems (see, e.g., [Ach98]). Concerning stress-constrained minimum weight problems, however, the situation is more complicated. Consider again the simple structure in Figure 1, and suppose that one wants, more naturally, to minimize the total weight subject to the stress constraints shown in the figure. ${ }^{2}$ The bar can sustain any stress with magnitude less than or equal to $\bar{\sigma}_{1}$ (specific for the bar material), while the cable can sustain no compressive stresses and no tensile stresses exceeding $\bar{\sigma}_{2}$ (specific

\footnotetext{
${ }^{2}$ This example will be studied in more detail in Section 4.2.4.
} 


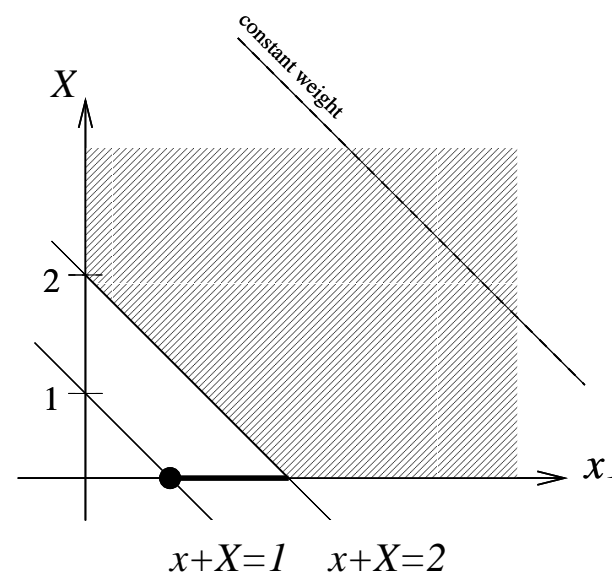

Figure 2: The admissible design domain. The optimal solution is at the black circle.

for the cable material). Figure 2 shows the feasible domain for the design variables $(x, X)$ after the state variables have been eliminated. Note that this domain consists of the union of a twodimensional, convex domain and a one-dimensional "spike" appended to it. As seen in the figure, the optimal design is located on the spike, and simply enforcing small strictly positive lower bounds on $x$ and $X$ will obviously change the feasible set in a discontinuous fashion; no matter how small lower design bound one uses, the distance to the desired optimal design will always be at least $1 / \sqrt{2}$.

Observations of this kind were done by Sved and Ginos as early as in 1968 [SvG68]. The problem is sometimes referred to as the "stress singularity phenomenon" or "singular topologies", cf., e.g., [Kir90, Roz01]. Two different perturbations which include $\varepsilon$-terms in the stress constraints were introduced by Svanberg [Sva94] and Cheng and Guo [ChG97]. Since it is of paramount importance to know if, and for which type of $\varepsilon$-perturbation, the restated sizing-like problem's design solutions converge to the set of optimal designs in the original problem statement as the parameter $\varepsilon$ approaches zero, it is the main theme of this paper to prove such continuity results, as well as the existence of optimal solutions, for several different problem classes. When justifying correct $\varepsilon$-perturbations the proofs rely heavily on the continuity properties of the mappings that provide the set of equilibrium states for given designs, including changes in the connectedness of the mechanical structure. These continuity results are interesting in their own right, and based on their generality we believe them to be useful also for applications other than those covered here.

We do not directly aim at providing methods for finding optimal solutions to the problems studied, but focus on justifying the optimization statements. Having proven that some sizing-like problem's optimal solutions are close to the desired ones, one can usually rely on the fact that the nested versions of sizing problems are often successfully solved by sequential convex or separable programming algorithms in conjunction with standard sensitivity analysis.

\section{$1.2 \quad$ Scope}

Two of the most natural and classical structural optimization problems are minimum compliance, or, equivalently, maximum stiffness, under a volume constraint, and minimum weight under stress constraints. We consider these problem classes in a discrete framework, that is, we assume that the state and design are specified by a finite number of variables, which is the case for example with 
trusses, where the state may be governed by a vector $u$ of nodal displacements and the design by a vector $x$ of bar volumes (or cross-sectional areas). We also treat a discretized continuous problem, namely the one that results from applying a finite element method (FEM) on the problem of minimizing the effective stress in an elastic continuum in plane state of stress. The objective is the minimum $L^{2}$-norm of the effective stress (in the sense of von Mises), subject to a constraint on the available amount of material volume. Different $\varepsilon$-perturbations are treated for all these three problem classes.

Frequently it is assumed that the elastic structure's state, for example nodal displacements and internal bar forces, is governed by a system of linear equations. In this work, we wish to allow for the modelling of mechanical contact and structures suspended by elements that can sustain only tensile forces, whence the equilibrium framework must be extended to affine variational inequalities (AVIs). These unilateral constraints appear in practice, for example, in machine elements such as press-fits and turbine blade roots, and for structures such as bridges suspended by elastic cables. When the state problem is governed by a variational inequality, the overall design problem is termed a mathematical program with equilibrium constraints (MPEC), cf. [LPR96], or a generalized bilevel optimization problem. For a general overview of optimization of structures subject to unilateral constraints, we refer to [HKP99b].

We also consider stochastic loads, that is, we allow for the applied loads to be random. For instance, a heavy weight hung by a crane could move in the wind in an unpredictable manner. The procedure is expected to lead to more robust optimal designs, since structures that are optimal for a single deterministic load can be very inefficient for slightly different loads, cf. [CPW01]. We assume that the load components are specified by a probability space, formulate functions affected by the stochastic data in terms of either expected values or worst-case scenarios, and then proceed by discretizing the corresponding sample space. Among other things, this may lead to a traditional multiple load-case formulation where a linear combination of the objective functions is used. The weights in this linear combination can be determined from the probability density function and the integration rule used to evaluate the expected value of the objective function. We remark that we could have considered a more general stochastic model which involves uncertainties in the other data of the problem, such as the initial distances to the rigid supports, which may be random due to manufacturing tolerances, the stiffness matrices, etc. We have chosen to study, from a mathematical standpoint, only one type of randomness, for two main reasons: first, external loads are probably the most natural choice of randomness in the model; second, the analysis of a more general stochastic model would be very similar.

\subsection{A preview and a gentle introduction to the techniques used}

In order to establish the topology optimization problems' transformation to sizing-like statements, continuity properties of the design-to-state mappings during topological changes need to be investigated. This requires in turn a proper description of the equilibrium relations for the states. The principle used must be expressed in such a way that possible topological changes are accounted for; consequently, it must distinguish between the active parts of the design, that is, those indices of the design variables which are nonzero, and those corresponding to holes or voids, that is, zero design variable values. It must also be amenable to sensitivity and perturbation analyses, wherein we investigate the changes in the equilibrium state to changes in the design. Perhaps somewhat surprisingly, neither does one equilibrium formulation suffice to reach the necessary results, nor does current sensitivity analysis cover all the perturbation and sensitivity results needed. We shall seek to explain below why this is the case, and to provide at the same time a gentle introduction to the results to follow in the later sections.

We will use three formulations of the equilibrium conditions for a given design $(x, X)$ : (a) the principle of minimum complementary energy, denoted $(\mathcal{C})_{(x, X)}$, which is described in terms of forces 
only; (b) the principle of minimum potential energy, denoted $(\mathcal{P})_{(x, X)}$, which is described in terms of displacements only; and (c) an AVI which is described in terms of forces and displacements simultaneously. The two first problems form, for a given design $(x, X)$, a primal-dual pair of quadratic programs, whose optimality conditions are exactly the AVI.

To each of these three formulations corresponds a main result on the continuity of the designto-state mapping. The first states that, given any convergent sequence of designs, and assuming the elastic energy remains bounded, the limit design possesses a state of equilibrium and the sequence of equilibrium forces converges to the limit design's equilibrium force (Theorem 3.1). (This is however not the case when the equilibrium principle is formulated in the displacement space.) The proof of the result utilizes the lower semicontinuity property of the extended real-valued energy function $\mathcal{E}$, and the formulation $(\mathcal{C})_{(x, X)}$. The other two continuity results are less general, as they are stated for particular design sequences where each element in the design vector decreases strictly monotonically to a fixed design variable value (and which vector is assumed to have a state in equilibrium). The total potential energy principle $(\mathcal{P})_{(x, X)}$ is in Theorem 3.2 utilized to establish that the sequence of displacements converges to the least-energy displacement among all possible equilibrium displacements. This is the only result presented which has been analyzed previously; it is in fact a special case of the perturbation technique known as the regularization of a nonstrictly convex program (e.g., [Bro66]); for this special caes, we felt however that an independent proof would be illustrative. The Theorems 3.3 and 3.4, finally, utilize the AVI formulation. The first result establishes that the convergence rate of the equilibria is at least linear. The proof is based on the recent development of the theory of error bounds for the solutions to AVIs and systems of linear inequalities, and on Theorem 3.2. The second result establishes that in the region where the design is strictly positive, the state is locally Lipschitz continuous in the design.

The design-to-state mapping is somewhat special, and in fact it is less "continuous" than what current sensitivity analysis presumes. The obstacle is the effect of changes in the topology. As the set of active indices in the design changes, the quadratic programs corresponding to the two equilibrium principles will have different numbers of constraints, in which case the feasible sets are not lower semicontinuous [the case of $(\mathcal{C})_{(x, X)}$ ], or the dimension of the null space of the objective's Hessian changes [the case of $(\mathcal{P})_{(x, X)}$ ]. (Sensitivity results are normally not formulated such that the number of constraints or dimension of the null space of a matrix are allowed to differ.) In an equivalent reformulation of $(\mathcal{C})_{(x, X)}$, the number of constraints is constant, but the objective is instead extended real-valued, with infinite values whenever a force is nonzero in an inactive design element. Although the energy functional is lower semicontinuous (lsc), it is not continuous at an equilibrium as it is not upper semicontinuous, which, again, precludes the use of existing analysis, such as Theorem 4.3.3 in [BGK+83]. (Present quantitative characterizations of continuity, e.g., upper- and lower-semicontinuity results, all require at least continuity of the objective function near the "reference point" jointly in the problem variables and parameters. It seems rather unlikely to be able to obtain a result applicable to a general class of optimization problems without this requirement. What helps us to be able to reach the sensitivity results sought are, in short, the favourable properties of the lsc function $(x, y) \mapsto x^{2} / y$ on $\mathbb{R} \times \mathbb{R}_{+}$, and the recent theory of error bounds for the solutions to AVIs.) The complementary energy problem's optimal solution is unique if a feasible solution exists. Restricted to a set where the energy is bounded, the design-to-force mapping is closed. The total potential energy principle can however in general have an unbounded solution set, due to the singularity of the stiffness matrix when some design variables are zero; further, the rank of this matrix changes dramatically with the index set of active design variables. To a sequence of equilibrium forces may correspond a divergent sequence of equilibrium displacements, if the limit design corresponds to a singular stiffness matrix. Thus, the designto-displacement mapping is not closed. The two quadratic programs are hence not amenable to traditional sensitivity analysis. The AVI is constructed such that a constraint enforces an inactive design element to have a zero force, so the problem is in some ways better posed. On the other 
hand, the sensitivity analysis of AVIs is weaker than for quadratic programs: the sensitivity analysis available is only local, unless the limit design is strictly positive, and can not be used to establish the existence of an equilibrium for a limit design.

Each of the three continuity results is applied in the results which then follow in Sections 4 and 5 , which are devoted to cable suspended trusses and finite element discretized sheets in contact, respectively. For each problem, we analyze the existence of optimal designs, and correct forms of $\varepsilon$-perturbation, for two problem statements: minimum compliance given a limited amount of material, and stress-constrained minimum weight. The compliance minimization problem is the easiest by far; the proofs use the design-to-force mapping's continuity as the main ingredient. The straightforward $\varepsilon$-perturbation mentioned earlier is sufficient, and the optimal design problem can be given several convex, or convex-concave, optimization formulations. The stress-constrained minimum weight problem is the most difficult. Existence relies on using design-to-force continuity; in the perturbation results, also the rate of convergence is needed, and in addition to setting the lower design bound to $\varepsilon$, we must introduce a term which converges to zero-faster than $\varepsilon$ but slower than $\varepsilon^{2}$-into the stress constraints. The problem where the effective stress in an elastic continuum is minimized is in character somewhat "in between" the other two problems in difficulty. Besides the design-to-force continuity, the displacements' convergence to the least-energy displacement is utilized. These results make it all the more clear that each of the three equilibrium principles studied have an important role to play.

To summarize, the rest of the paper is organized as follows. Section 2 deals with the three principles of equilibrium: minimum of complementary energy, minimum total potential energy, and an AVI expressed in all state variables simultaneously. Section 3 states and proves several propositions on the continuity properties of the state variables with respect to changes in the design variables, including changes in topology. The following two sections deal with interesting instances of structural optimization problems. Section 4 accounts for cable suspended trusses, minimum compliance as well as stress constrained minimum weight. Section 5 treats the FEdiscretized sheet problem where the $L^{2}$-norm of the effective stress is minimized. Finally, Section 6 includes some remarks and comments on interesting further research.

\section{The equilibrium problem}

The structure is assumed to consist of at most $m$ parts such as bars or finite elements. The material volume allocated at part $i$ is described by $x_{i}, i=1, \ldots, m$. Clearly, $x_{i} \geq 0$ holds, and $x_{i}=0$ is interpreted as a structural void. We denote the set of present (or, active) parts of the structure by the index set

$$
\mathcal{I}(x):=\left\{i \mid x_{i}>0\right\} \subseteq\{1, \ldots, m\} .
$$

The structure is further assumed to consist of nodes, the displacements of which are collected in a (column) vector $u \in \mathbb{R}^{n}$ (note: prescribed zero-displacements are removed). The deformation of each (present) part is described by $s c$ strain components, collected in a vector $\varepsilon_{i}$ for $i=1, \ldots, m$. This strain is connected to the displacement through the relation

$$
\varepsilon_{i}=B_{i} u, \quad i \in \mathcal{I}(x),
$$

where $B_{i} \in \mathbb{R}^{s c \times n}$ is a kinematic transformation matrix. The stress state of each present part is described by $\sigma_{i} \in \mathbb{R}^{s c}$, and is assumed to be related to the strain according to Hooke's generalized law:

$$
\sigma_{i}=E \varepsilon_{i}, \quad i \in \mathcal{I}(x),
$$


where $E \in \mathbb{R}^{s c \times s c}$ is the symmetric and positive definite matrix of elasticity constants. We define the force-like variable $s_{i}$ as

$$
s_{i}=x_{i} \sigma_{i}, \quad i \in \mathcal{I}(x),
$$

which in fact has unit (force $\times$ length). (For a bar it is simply the bar force times the bar length.) We set $D_{i}(x)=x_{i} E$, and then (1)-(3) give

$$
s_{i}=D_{i}(x) B_{i} u, \quad i \in \mathcal{I}(x) .
$$

External forces (including forces due to unilateral contact and cables) are represented by a vector $F \in \mathbb{R}^{n} ;$ static force equilibrium is governed by

$$
F=\sum_{i \in \mathcal{I}(x)} B_{i}^{T} s_{i} .
$$

Note that, if we define the structural stiffness matrix as

$$
K(x)=\sum_{i=1}^{m} x_{i} B_{i}^{T} E B_{i},
$$

then (4) and (5) yield

$$
F=K(x) u \text {. }
$$

We assume that there are sufficiently many prescribed zero-displacements for $K\left(1_{m}\right)$ to be positive definite. (Here, $1_{m}$ denotes the $m$-vector of ones.)

\subsection{Special case 1: Linear triangular finite elements}

In this particular case, we consider a plane structure, $s c=3$ and strains $\varepsilon_{i}=\left(\varepsilon_{x}, \varepsilon_{y}, \gamma_{x y}\right)^{T}$, stresses $\sigma_{i}=\left(\sigma_{x}, \sigma_{y}, \tau_{x y}\right)^{T}$, and, assuming plane stress and an isotropic elastic body,

$$
E=\frac{E_{0}}{1-\nu^{2}}\left(\begin{array}{ccc}
1 & \nu & 0 \\
\nu & 1 & 0 \\
0 & 0 & \frac{1-\nu}{2}
\end{array}\right),
$$

where $\nu \in(-1,1 / 2)$ is Poisson's ratio and $E_{0}>0$ is Young's modulus. Let the set $\eta_{i}$ contain the node numbers for the nodes belonging to element $i$. If we define matrices

$$
B_{A}=\left(\begin{array}{cc}
\frac{\partial N_{A}}{\partial x_{1}} & 0 \\
0 & \frac{\partial N_{A}}{\partial x_{2}} \\
\frac{\partial N_{A}}{\partial x_{2}} & \frac{\partial N_{A}}{\partial x_{1}}
\end{array}\right),
$$

where $N_{A}$ are shape functions, then the $B_{i}$ 's are constructed so that

$$
\varepsilon_{i}=\sum_{A \in \eta_{i}} B_{A} u_{A}=B_{i} u,
$$

where $u_{A} \in \mathbb{R}^{2}$ is the displacement at node $A$.

Based on the different stress components in a finite element one can calculate a single effective stress according to

$$
\sigma_{i}^{e}=\sqrt{\sigma_{i}^{T} M \sigma_{i}},
$$


where, in the case of von Mises effective stress,

$$
M=\left(\begin{array}{ccc}
1 & -\frac{1}{2} & 0 \\
-\frac{1}{2} & 1 & 0 \\
0 & 0 & 3
\end{array}\right)
$$

\subsection{Special case 2: Truss structures}

This particular case involves a set of bars in one, two or three dimensions. We have $s c=1, \sigma_{i}$ is simply the stress in the bar, and $\varepsilon_{i}$ the strain. The matrix $E$ is Young's modulus of the bar, and

$$
D_{i}=x_{i} E, \quad s_{i}=x_{i} \sigma_{i} .
$$

Hence, $s_{i}$ is the bar force times its length, $B_{i}$ is $1 / L_{i} \bar{\gamma}_{i}^{T}$, where $L_{i}$ is the bar's length, and $\bar{\gamma}_{i}$ contains the bar's direction cosines.

The effective stress in a bar is the absolute value of the single stress value; hence, $\sigma_{i}^{e}=$ $\sqrt{\sigma_{i}^{T} M \sigma_{i}}=\left|\sigma_{i}\right|$ holds, where here $M=1$ holds.

\subsection{The unilateral constraints}

Suppose we have unilateral rigid supports that cannot be penetrated by the nodes of the structure. Then, these unilateral constraints can be formulated as

$$
C_{1} u \leq g_{1}
$$

where $C_{1} \in \mathbb{R}^{r_{1} \times n}$ is a kinematic transformation matrix, and $g_{1} \in \mathbb{R}^{r_{1}}$ is the vector of the initial gaps. If we let $\lambda \in \mathbb{R}^{r_{1}}$ be the vector of contact forces, then the relations

$$
\lambda \geq 0, \quad \lambda^{T}\left(C_{1} u-g_{1}\right)=0
$$

reflect the facts that the contact is non-adhesive and that contact forces do not develop at a distance. We shall assume that each node is subject to not more than one contact condition (or, more generally, that if there are more than one contact condition for one node then they are orthogonal), whence $C_{1} C_{1}^{T}$ equals the $r_{1} \times r_{1}$ identity matrix. (We refer to this as $C_{1}$ being quasi-orthogonal.)

Suppose now also that there are at most $r_{2}$ cables (or, ropes), the ends of which are attached to nodes of the structure and (possibly) also suspended at rigid foundations. The $j$ th cable's volume is denoted by $X_{j}$, and similarly to $\mathcal{I}(x)$ we define

$$
\mathcal{J}(X):=\left\{j \mid X_{j}>0\right\} \subseteq\left\{1, \ldots, r_{2}\right\} .
$$

We let $e_{j}$ be the cable's elongation, $\left(g_{2}\right)_{j}$ its initial slack, and $S_{j}$ its tensile force. Then, the behavior of the cables can be described by

$$
\gamma_{j}^{T} u-e_{j}-\left(g_{2}\right)_{j} \leq 0, \quad S_{j} \geq 0, \quad\left(\gamma_{j}^{T} u-e_{j}-\left(g_{2}\right)_{j}\right) S_{j}=0, \quad j \in \mathcal{J}(X),
$$

where $\gamma_{j}$ is a vector which contains the unit vector of cable $j$ (in the same way as for a bar element). The cables' stiffness constants are

$$
k_{j}(X)=\frac{X_{j} E_{c}}{L_{j}^{2}}, \quad j \in \mathcal{J}(X)
$$


where $E_{c}>0$ is Young's modulus for the cable material and $L_{j}>0$ the cable lengths. Therefore, the cable elasticity is modelled by

$$
S_{j}=k_{j}(X) e_{j}, \quad j \in \mathcal{J}(X),
$$

(and hence $S_{j} \geq 0$ if and only if $e_{j} \geq 0$ ). We interpret (10) and (12) as follows: if $S_{j}=0$, then by (12), $e_{j}=0$, and what is left of (10) is $\gamma_{j}^{T} u \leq\left(g_{2}\right)_{j}$ which asserts that the elongation of the straight line between the cable's end points cannot exceed the initial slack. If, on the other hand, $S_{j}>0$, then (10) states that $\gamma_{j}^{T} u-e_{j}=\left(g_{2}\right)_{j}$, that is, the cable elongation equals the elongation of the straight line between the cable's end points minus the initial slack.

The cable forces are directed along the direction of the cables, defined through the vector $\gamma_{j}$; we presume frictionless contact, whence the directions of contact forces are known (namely orthogonally to the unilateral supports). Hence, if $f \in \mathbb{R}^{n}$ denotes external prescribed forces (different from cable and contact forces), static equilibrium [see (5)] is governed by

$$
C_{1}^{T} \lambda+\sum_{i \in \mathcal{I}(x)} B_{i}^{T} s_{i}+\sum_{j \in \mathcal{J}(X)} S_{j} \gamma_{j}=f,
$$

since

$$
F=f-C_{1}^{T} \lambda-\sum_{j \in \mathcal{J}(X)} S_{j} \gamma_{j}
$$

Given a structure and cable design $(x, X)$, the overall equilibrium problem can now be summarized as follows: For all $i \in \mathcal{I}(x)$ and $j \in \mathcal{J}(X)$, find nodal displacements $u$, cable elongations $e_{j}$, contact forces $\lambda$, cable forces $S_{j}$ and internal structure forces $s_{i}$ such that (4), (8)-(10), and (12)-(13) hold.

\subsection{Energy principles}

\subsubsection{Minimum complementary energy}

The principle of minimum of complementary energy states that among all force distributions that satisfy static force equilibrium [that is, (5)], the one present in equilibrium (if any), is one which minimizes the elastic energy of the structure. In parts of the structure where $x_{i}=0$ or $X_{j}=0$ holds, elastic energy cannot be stored. Using the notation $\mathcal{I}(x)$ and $\mathcal{J}(X)$ for positive elements $x_{i}$ and $X_{j}$, respectively, and $s_{\mathcal{I}(x)}$ and $S_{\mathcal{J}(X)}$ for the corresponding sub-vectors, we can then state the elastic energy minimization problem as follows:

$$
(\mathcal{C})_{(x, X)}\left\{\begin{aligned}
\min _{\left(s_{\mathcal{I}(x)}, S_{\mathcal{J}(X)}, \lambda\right)} & \mathcal{E}_{\mathcal{I}, \mathcal{J}}(x, X, s, S, \lambda):=\frac{1}{2} \sum_{i \in \mathcal{I}(x)} \frac{s_{i}^{T} E^{-1} s_{i}}{x_{i}}+g_{1}^{T} \lambda \\
& +\sum_{j \in \mathcal{J}(X)}\left(\frac{\left(L_{j} S_{j}\right)^{2}}{2 E_{c} X_{j}}+\left(g_{2}\right)_{j} S_{j}\right), \\
\text { s.t. } & \left\{\begin{array}{r}
C_{1}^{T} \lambda+\sum_{i \in \mathcal{I}(x)} B_{i}^{T} s_{i}+\sum_{j \in \mathcal{J}(X)} S_{j} \gamma_{j}=f, \\
\lambda \geq 0, \\
S_{\mathcal{J}(X)} \geq 0 .
\end{array}\right.
\end{aligned}\right.
$$

In the below result, we use the notions that a real-valued function, say $\varphi: \mathbb{R}^{n} \mapsto \mathbb{R} \cup\{+\infty\}$, is coercive (with respect to a set $Y$ ) if $Y$ is bounded or $\lim _{\left\|x^{t}\right\| \rightarrow \infty, x^{t} \in Y} \varphi\left(x^{t}\right)=\infty$, and that $\varphi$ is lower semicontinuous (lsc) if for any $x \in \mathbb{R}^{n}, \liminf _{y \rightarrow x} \varphi(y) \geq \varphi(x)$. 
Theorem 2.1 (Existence of optimal solutions to $\left.(\mathcal{C})_{(x, X)}\right)$. Suppose the feasible set of the problem $(\mathcal{C})_{(x, X)}$ is nonempty. Then, there exists a unique optimal solution to the problem $(\mathcal{C})_{(x, X)}$.

Proof. The objective function of $(\mathcal{C})_{(x, X)}$ is coercive and lsc. The former follows for $\left(s_{\mathcal{I}(x)}, S_{\mathcal{J}(X)}\right)$ immediately, and for $\lambda$ we note that by the quasi-orthogonality of $C_{1}, \lambda$ is uniquely determined by them. Lower semicontinuity follows from continuity. Since $\mathcal{E}_{\mathcal{I}, \mathcal{J}}$ is strictly convex in those variables, also uniqueness follows.

We investigate the optimality conditions for this problem. We introduce $u$ as the Lagrange multipliers for the equality constraints. The stationarity conditions for the Lagrangian then become the following: stationarity with respect to $s$ gives (4); through the definition (11) we derive (10) and (12) as the stationarity conditions with respect to $S \geq 0$; stationarity with respect to $\lambda \geq 0$ yields (8) and (9); finally, stationarity with respect to $u$ of course gives us (13). Summarizing, then, the conditions which characterize the minimal complementary energy are precisely the conditions (4), (8)-(10), and (12)-(13), discussed from a mechanical standpoint in the previous section.

\subsubsection{Minimum total potential energy}

We will next state and investigate a principle of minimum potential energy. Given a design $(x, X)$ the problem is the following:

$$
(\mathcal{P})_{(x, X)}\left\{\begin{array}{cl}
\min _{\left(u, e_{\mathcal{J}(\mathcal{X})}\right)} & \frac{1}{2} \sum_{i \in \mathcal{I}(x)} x_{i} u^{T} B_{i}^{T} E B_{i} u+\frac{1}{2} \sum_{j \in \mathcal{J}(X)} \frac{E_{c} X_{j}}{L_{j}^{2}} e_{j}^{2}-f^{T} u, \\
\text { s.t. } & \left\{\begin{array}{c}
C_{1} u \leq g_{1}, \\
\gamma_{j}^{T} u-e_{j} \leq\left(g_{2}\right)_{j}, \quad j \in \mathcal{J}(X) .
\end{array}\right.
\end{array}\right.
$$

Investigating its optimality conditions, we introduce $\lambda \geq 0$ and $S_{j} \geq 0, j \in \mathcal{J}(X)$, as the Lagrange multipliers for the inequality constraints. Pursuing, as for the problem $(\mathcal{C})_{(x, X)}$, the stationarity conditions for the resulting Lagrangian reformulation, we obtain the following. We first note that although $s$ does not enter into this problem, we will use (4) as its definition. Then, stationarity with respect to $u$ yields (13); stationarity with respect to $e$ gives (12); stationarity with respect to $\lambda \geq 0$ gives (8) and (9); finally, stationarity with respect to $S \geq 0$ gives (10). So, to summarize, the characterization of the minimum potential energy is, again, the conditions (4), (8)-(10), and (12)-(13), discussed from a mechanical standpoint in the previous section.

This development also establishes that the problems $(\mathcal{C})_{(x, X)}$ and $(\mathcal{P})_{(x, X)}$ constitute equivalent, primal-dual pairs of convex quadratic programs, since they have the same optimality conditions. This means that if there exists an optimal solution to $(\mathcal{C})_{(x, X)}$, then there are optimal solutions to $(\mathcal{P})_{(x, X)}$, and, conversely, if there is at least one optimal solution to $(\mathcal{P})_{(x, X)}$, then $(\mathcal{C})_{(x, X)}$ is uniquely solvable.

In the optimal solution to these problems, the values of the variables $s_{\mathcal{I} \backslash \mathcal{I}(x)}$ and $(S, e)_{\mathcal{J} \backslash \mathcal{J}(X)}$ are unspecified. (This is a direct effect of the way in which the primal-dual pair of equilibrium problems were stated, as these variables are not present in their formulations or in their Lagrangianbased optimality conditions.) This is, however, a drawback when we want to consider existence, continuity and other sensitivity issues for varying values of $(x, X)$ [and consequently for varying index sets $\mathcal{I}(x)$ and $\mathcal{J}(X)$ ], in particular as a subset of their elements tend to zero. In order to state a complete set of equilibrium conditions, containing all the variables, we shall next formulate an affine variational inequality problem which embraces the conditions (4), (8)-(10), and (12)-(13). The idea is to introduce the conditions (4) and (12) explicitly into the formulation, for the entire sets of variables, and not just for the index sets $\mathcal{I}(x)$ and $\mathcal{J}(X)$, whereby we explicitly account for 
the active parts of the structure by forcing zero elements in $(x, X)$ to correspond to zero elements in $(s, S)$. Mechanically speaking, the only possible force in a void is zero.

\subsubsection{Equilibrium characterization as an affine variational inequality}

Let $Q$ be a matrix in $\mathbb{R}^{p \times p}, q$ a vector in $\mathbb{R}^{p}$ and $Y$ a polyhedral subset of $\mathbb{R}^{p}$. The affine variational inequality (AVI) problem associated with this data is to find $y^{*} \in Y$ such that

$$
\left[Q y^{*}+q\right]^{T}\left(y-y^{*}\right) \geq 0, \quad y \in Y .
$$

(In case $Q$ is symmetric, this variational inequality constitutes the necessary conditions for $y^{*}$ to be a local minimum point of the function $y \mapsto \frac{1}{2} y^{T} Q y+q^{T} y$ over the set $Y$.) We denote this problem by $\operatorname{AVI}(q, Q, Y)$.

We now state the equilibrium conditions for forces and displacements as an AVI. To this end, we first define $C_{2}$ as the $r_{2} \times n$ matrix of the vectors $\gamma_{j}, B$ as the $(m \cdot s c) \times n$ matrix created by stacking the matrices $B_{i}$ on top of each other, $s$ as the $m \cdot s c$ vector created by stacking the vectors $s_{i}$ on top of each other, $D(x)$ as the $(m \cdot s c) \times(m \cdot s c)$ block-diagonal matrix created by placing the matrices $D_{i}(x)$ along the diagonal, and, finally, $k(X)$ as the $r_{2} \times r_{2}$ diagonal matrix with diagonal elements $k_{j}(X)$. Let

$$
y:=\left(\begin{array}{c}
u \\
e \\
s \\
S \\
\lambda
\end{array}\right), \quad Q:=\left(\begin{array}{ccccc}
0 & 0 & B^{T} & C_{2}^{T} & C_{1}^{T} \\
D(x) B & 0 & -I & 0 & 0 \\
0 & k(X) & 0 & -I & 0 \\
-C_{2} & I & 0 & 0 & 0 \\
-C_{1} & 0 & 0 & 0 & 0
\end{array}\right), \quad \text { and } \quad q:=\left(\begin{array}{c}
-f \\
0 \\
0 \\
g_{2} \\
g_{1}
\end{array}\right),
$$

and $Y:=\mathbb{R}^{n} \times \mathbb{R}^{r_{2}} \times \mathbb{R}^{m \cdot s c} \times \mathbb{R}_{+}^{r_{2}} \times \mathbb{R}_{+}^{r_{1}}$. (Note: $\mathbb{R}_{+}$denotes the set of nonnegative reals, whereas $\mathbb{R}_{++}$, to be used later, denotes the set of strictly positive reals.)

It is easy to check that the AVI with this data is a statement of the conditions (13), (4), (12), $(10),(8),(9)$, in that order, where now the conditions are stated over all the variables.

For the AVI given by (14), (15) we next establish an elementary result on the closedness property of its solution set when viewing $(x, X)$ as parameters. To this end, we shall introduce the new notation AVI $(q, Q(x, X), Y)$ and SOL $(q, Q(x, X), Y)$ to denote the AVI problem (14), (15) and its solution set for a given pair $(x, X)$.

Theorem 2.2 (Closedness of the mapping $(x, X) \mapsto \mathrm{SOL}(q, Q(x, X), Y))$. The mapping $(x, X) \mapsto$ $\operatorname{SOL}(q, Q(x, X), Y)$ is closed on $\mathbb{R}^{m} \times \mathbb{R}^{r_{2}}$.

Proof. Consider a sequence $\mathbb{R}^{m} \times \mathbb{R}^{r_{2}} \supset\left\{\left(x^{t}, X^{t}\right)\right\} \rightarrow\left(x^{*}, X^{*}\right)$, and an arbitrary sequence $\left\{y^{t}\right\}$ fulfilling $y^{t} \in \operatorname{SOL}\left(q, Q\left(x^{t}, X^{t}\right), Y\right)$ for all $t$. Suppose that the latter sequence has a limit point, $y^{*}$. Closedness amounts to having $y^{*} \in \operatorname{SOL}\left(q, Q\left(x^{*}, X^{*}\right), Y\right)$. In order to establish this inclusion, consider, for a fixed vector $y \in Y$ in the AVI given by (14), (15), the sequence of solutions over $t$. Then, noting that $Q(\cdot, \cdot)+q$ is continuous in $(x, X)$, the result clearly follows.

The reader is advised not to conclude that there always exist limit states for a limit design: the assumption of boundedness of the sequence of states, made in the proof of the above lemma, is crucial. Subsequently, we shall illustrate in detail that there are indeed cases where limit displacements do not exist (see Section 3.1.2).

Further, the above result can not be used to claim that a bounded sequence of forces accumulate at equilibrium forces for a limit design. For this to be true, the energy needs to remain bounded, cf. Theorem 3.1 and Corollary 3.1. 


\section{Continuity of design-to-state mappings}

We now investigate the behavior of the equilibrium states $(u, e),(s, S)$, and $\lambda$ as functions of the designs $(x, X)$. In particular, we are interested in the continuity of sequences of equilibrium states as a sequence of designs tends to a limit. This will be very useful in the analysis of $\varepsilon$-perturbation schemes, wherein small but positive lower design bounds are used, and which may subsequently be allowed to tend to zero.

\subsection{Design-to-force}

\subsubsection{Theoretical results}

We first consider the conditions under which a limit state exists for a design. We begin by two useful lemmas.

Lemma 3.1 (Lower semicontinuity of a convex function). Let the convex function $\varphi: \mathbb{R} \times \mathbb{R}_{+} \mapsto$ $\mathbb{R}_{+} \cup\{+\infty\}$ be defined by

$$
\varphi(x, y)= \begin{cases}x^{2} / y, & \text { if } y>0 \\ +\infty, & \text { if } x \neq 0 \text { and } y=0 \\ 0, & \text { if } x=y=0\end{cases}
$$

Then, $\varphi$ is lsc on $\mathbb{R} \times \mathbb{R}_{+}$, and $\varphi(x, \cdot)$ is continuous on $\mathbb{R}_{+}$for any $x \in \mathbb{R}$.

Proof. The analysis of this function is similar to that in Rockafellar [Roc70, p. 83], which, however, concerns its lsc property over a larger domain. Let $\mathbb{R} \supset\left\{x^{t}\right\} \rightarrow x$ and $\mathbb{R}_{+} \supset\left\{y^{t}\right\} \rightarrow y$. We need to show that (i) $\liminf _{t \rightarrow \infty} \varphi\left(x^{t}, y^{t}\right) \geq \varphi(x, y)$, and (ii) $\lim _{t \rightarrow \infty} \varphi\left(x, y^{t}\right)=\varphi(x, y)$.

Consider first the case $(x, y)=(0,0)$. Here, $\varphi(x, y)=0 \leq \varphi\left(x^{t}, y^{t}\right)$, so (i) follows immediately. Also (ii) holds since $\varphi\left(0, y^{t}\right)=0=\varphi(x, y)$.

The second case is $x \neq 0$ and $y=0$. Here, $\varphi(x, y)=+\infty$. Since $\left|x^{t}\right| \geq c>0$ for all sufficiently large $t$, one has either that $\varphi\left(x^{t}, y^{t}\right) \geq c^{2} / y^{t}$ (if $y^{t}>0$ ) or $\varphi\left(x^{t}, y^{t}\right)=+\infty$ (if $y^{t}=0$ ). In either case, (i) follows, and (ii) follows similarly.

The third case is $y>0$. Here, $\varphi\left(x^{t}, y^{t}\right)=\left(x^{t}\right)^{2} / y^{t}$ for all sufficiently large $t$, so both (i) and (ii) holds. This completes the proof.

We next apply this result to our energy functional. For the sake of a subsequent discussion, we will state the following result for a more general energy function.

Lemma 3.2 (Lower semicontinuity of an energy functional). Let $M$ be an arbitrary symmetric and positive definite $s c \times s c$ matrix, and $M^{1 / 2}$ an arbitrary symmetric and positive definite square root. With $\varphi$ being the function in Lemma 3.1, the function

$$
\mathcal{E}^{M}(x, X, s, S, \lambda):=\frac{1}{2} \sum_{i=1}^{m} \sum_{k=1}^{s c} \varphi\left(\left(M^{1 / 2} s_{i}\right)_{k}, x_{i}\right)+g_{1}^{T} \lambda+\sum_{j=1}^{r_{2}}\left(\frac{L_{j}^{2}}{2 E_{c}} \varphi\left(S_{j}, X_{j}\right)+\left(g_{2}\right)_{j} S_{j}\right)
$$

is convex and lsc on $\mathbb{R}_{+}^{m} \times \mathbb{R}_{+}^{r_{2}} \times \mathbb{R}^{m \cdot s c} \times \mathbb{R}^{r_{2}} \times \mathbb{R}^{r_{1}}$, and $\mathcal{E}^{M}(\cdot, \cdot, s, S, \lambda)$ is continuous on $\mathbb{R}_{+}^{m} \times \mathbb{R}_{+}^{r_{2}}$ for any $(s, S, \lambda)$ in $\mathbb{R}^{m \cdot s c} \times \mathbb{R}^{r_{2}} \times \mathbb{R}^{r_{1}}$.

Proof. The result follows from Lemma 3.1 and the fact that the sum of convex, lsc functions is convex and lsc (e.g., [Roc70, Theorem 9.3]). 
Note that with $M=E^{-1}, \mathcal{E}^{M}$ agrees with the energy functional $\mathcal{E}_{\mathcal{I}, \mathcal{J}}$ appearing in $(\mathcal{C})_{(x, X)}$ at arguments where it is finite. Note further that if we want the energy functional to stay finite also when considering the case where some design variables in $(x, X)$ are zero, then we must enforce the corresponding force elements in $(s, S)$ to be equal to zero. [This corresponds to applying the definitions (4) and (12).]

We shall, however, henceforth consider formulations of the equilibrium problems where all design and state variables are present, since we believe it to be more appropriate when analyzing existence and sensitivity questions. So, when referring to the problems $(\mathcal{C})_{(x, X)}$ and $(\mathcal{P})_{(x, X)}$, we shall (implicitly) presume that all elements of $(x, X)$ are present both in the objective function and in the constraints, whether they are active (all having positive values) or not. Moreover, the vectors $s_{\mathcal{I} \backslash \mathcal{I}(x)}$ and $S_{\mathcal{J} \backslash \mathcal{J}(X)}$ are included and, whenever the energy is finite, forced to zero. The effect on the problem $(\mathcal{C})_{(x, X)}$ is twofold, the first resulting in a simplification, the second in a slight complication: (1) the feasible set, henceforth denoted by $\mathcal{F}^{\mathcal{C}}$, does no longer depend on the design $(x, X)$; and $(2)$ the energy functional, $\mathcal{E}$, is an extended real-valued function, possibly taking on infinite values where one or more design variable values are zero. For the problem $(\mathcal{P})_{(x, X)}$, the effect is also twofold: (1) the feasible set, denoted by $\mathcal{F}^{\mathcal{P}}$, is not dependent on the design; and (2) the elements $e_{\mathcal{J} \backslash \mathcal{J}(X)}$ may be specified to arbitrary values, but large enough so that $\gamma_{j}^{T} u-e_{j}-\left(g_{2}\right)_{j} \leq 0$ holds for all $j \in \mathcal{J} \backslash \mathcal{J}(X)$.

From now on, whenever referring to a functional, like $\mathcal{E}$, where only the active design elements are present, we shall write $\mathcal{E}_{\mathcal{I}, \mathcal{J}}$. (Further, whenever $M=E^{-1}$ in the energy functional, the superscript ${ }^{M}$ will be suppressed.)

Recall that a function $\varphi: \mathbb{R}^{n} \mapsto \mathbb{R} \cup\{+\infty\}$ is proper if $\varphi(x)<+\infty$ holds for at least one $x \in \mathbb{R}^{n}$ and $\varphi(x)>-\infty$ holds for all $x \in \mathbb{R}^{n}$. We also refer to a function $\varphi$ as being proper with respect to a set $Y$, then meaning that the function $\varphi+\delta_{Y}$ is proper, where $\delta_{Y}$ is the indicator function of the set $Y\left(\delta_{Y}(x)=0\right.$ for $x \in Y ; \delta_{Y}(x)=+\infty$ for $\left.x \notin Y\right)$.

Theorem 3.1 (Existence of a force equilibrium). Let $\left\{\left(x^{t}, X^{t}\right)\right\}$ be a nonnegative sequence of designs, converging to $(x, X)$. Suppose that $\left\{\left(s^{t}, S^{t}, \lambda^{t}\right)\right\}$ is the corresponding sequence of optimal solutions to $(\mathcal{C})_{\left(x^{t}, X^{t}\right)}$, and assume that the sequence of energies is bounded, that is, that $\mathcal{E}\left(x^{t}, X^{t}, s^{t}, S^{t}, \lambda^{t}\right) \leq c<\infty$ for all $t$. Then, there exists a unique optimal solution $(s, S, \lambda)$ to $(\mathcal{C})_{(x, X)}$, and $\left\{\left(s^{t}, S^{t}, \lambda^{t}\right)\right\} \rightarrow(s, S, \lambda)$.

Proof. That the sequence $\left\{\left(s^{t}, S^{t}, \lambda^{t}\right)\right\}$ is bounded follows by the coercivity of $\mathcal{E}$, which is uniform with respect to $(x, X)$ [cf. the proof of Theorem 2.1], and the boundedness of $\left\{\left(x^{t}, X^{t}\right)\right\}$, together with the assumed existence of $c$. Let $(s, S, \lambda)$ be an arbitrary limit point of this sequence. The lsc property of $\mathcal{E}$ (cf. Lemma 3.2) and the assumption yields that

$$
\mathcal{E}(x, X, s, S, \lambda) \leq \liminf _{t \rightarrow \infty} \mathcal{E}\left(x^{t}, X^{t}, s^{t}, S^{t}, \lambda^{t}\right) \leq c<\infty,
$$

so we can conclude that $(s, S, \lambda) \in \mathcal{F}^{\mathcal{C}}$ and that $\mathcal{E}(x, X, \cdot, \cdot, \cdot)$ is proper with respect to $\mathcal{F}^{\mathcal{C}}$; moreover, by Theorem 2.1, the optimal solution to $(\mathcal{C})_{(x, X)}$ is unique.

Let now $(\bar{s}, \bar{S}, \bar{\lambda}) \in \mathcal{F}^{\mathcal{C}}$. Then, from $\mathcal{E}\left(x^{t}, X^{t}, s^{t}, S^{t}, \lambda^{t}\right) \leq \mathcal{E}\left(x^{t}, X^{t}, \bar{s}, \bar{S}, \bar{\lambda}\right)$ for all $t$ follows

$$
\begin{aligned}
\mathcal{E}(x, X, s, S, \lambda) & \leq \liminf _{t \rightarrow \infty} \mathcal{E}\left(x^{t}, X^{t}, s^{t}, S^{t}, \lambda^{t}\right) \\
& \leq \lim _{t \rightarrow \infty} \mathcal{E}\left(x^{t}, X^{t}, \bar{s}, \bar{S}, \bar{\lambda}\right) \\
& =\mathcal{E}(x, X, \bar{s}, \bar{S}, \bar{\lambda}),
\end{aligned}
$$

where the equality follows by the continuity of $\mathcal{E}(\cdot, \cdot, \bar{s}, \bar{S}, \bar{\lambda})$ [cf. Lemma 3.2]. It follows that $(s, S, \lambda)$ is optimal in $(\mathcal{C})_{(x, X)}$. Therefore, $(s, S, \lambda)$ must also be the only limit point of the sequence $\left\{\left(s^{t}, S^{t}, \lambda^{t}\right)\right\}$. 
As remarked after Theorem 2.2, the projection of $\operatorname{SOL}(q, Q(x, X), Y)$ onto the subspace of forces $(s, S, \lambda)$, that is, the result of eliminating the displacements, leads not to a closed mapping. Under the condition that the energy remains bounded, however, we can establish such a result. To this end, we define, respectively, the graph of the equilibrium mapping in terms of forces only, and the lower level set of the energy function $\mathcal{E}$, as follows:

$$
\begin{aligned}
\operatorname{gr} \mathcal{S} & :=\{(x, X, s, S, \lambda) \mid(u, e, s, S, \lambda) \text { solves }(14) \text { and }(15)\} \\
L_{\nu}^{\mathcal{E}} & :=\{(x, X, s, S, \lambda) \mid \mathcal{E}(x, X, s, S, \lambda) \leq \nu\}
\end{aligned}
$$

Corollary 3.1 (Closedness of the force equilibrium mapping). Let $\nu \in \mathbb{R}$. Then, the set gr $\mathcal{S} \cap L_{\nu}^{\mathcal{E}}$ is closed.

Proof. Let $\nu \in \mathbb{R}$. Consider a sequence $\left\{\left(x^{t}, X^{t}, s^{t}, S^{t}, \lambda^{t}\right)\right\} \subset \operatorname{gr} \mathcal{S} \cap L_{\nu}^{\mathcal{E}}$ with $\left\{\left(x^{t}, X^{t}\right)\right\} \subset$ $\mathbb{R}_{+}^{m} \times \mathbb{R}_{+}^{r_{2}}$, and assume that $\left\{\left(x^{t}, X^{t}, s^{t}, S^{t}, \lambda^{t}\right)\right\} \rightarrow(x, X, s, S, \lambda)$. The lsc property of $\mathcal{E}$ ensures that $\mathcal{E}(x, X, s, S, \lambda) \leq \liminf _{t \rightarrow \infty} \mathcal{E}\left(x^{t}, X^{t}, s^{t}, S^{t}, \lambda^{t}\right) \leq \nu$. So, $(x, X, s, S, \lambda) \in L_{\nu}^{\mathcal{E}}$. By Theorem $3.1,(s, S, \lambda)$ is moreover the optimal solution to $(\mathcal{C})_{(x, X)}$, whence $(x, X, s, S, \lambda) \in \operatorname{gr} \mathcal{S}$ also holds.

A result of a character similar to that of Theorem 3.1 will be useful in the subsequent analysis.

Corollary 3.2 (Convergence of equilibrium forces). Let $(x, X)$ be a nonnegative design for which $\mathcal{E}(x, X, \cdot, \cdot, \cdot)$ is proper with respect to $\mathcal{F}^{\mathcal{C}}$, and let $(s, S, \lambda)$ be the optimal solution to the problem $(\mathcal{C})_{(x, X)}$. Let $\left\{\left(x^{t}, X^{t}\right)\right\}$ be a sequence of nonnegative designs which converges to $(x, X)$, and suppose that $\left\{\left(s^{t}, S^{t}, \lambda^{t}\right)\right\}$ is the corresponding sequence of optimal solutions to $(\mathcal{C})_{\left(x^{t}, X^{t}\right)}$. Then, $\left\{\left(s^{t}, S^{t}, \lambda^{t}\right)\right\} \rightarrow(s, S, \lambda)$.

Proof. The relations

$$
\begin{aligned}
\limsup _{t \rightarrow \infty} \mathcal{E}\left(x^{t}, X^{t}, s^{t}, S^{t}, \lambda^{t}\right) & \leq \lim _{t \rightarrow \infty} \mathcal{E}\left(x^{t}, X^{t}, s, S, \lambda\right) \\
& =\mathcal{E}(x, X, s, S, \lambda)<\infty
\end{aligned}
$$

follow from the optimality of $\left(s^{t}, S^{t}, \lambda^{t}\right)$ in the problem $(\mathcal{C})_{\left(x^{t}, X^{t}\right)}$ and the continuity of $\mathcal{E}(\cdot, \cdot, s, S, \lambda)$ [cf. Lemma 3.2]. Therefore, the sequence $\left\{\mathcal{E}\left(x^{t}, X^{t}, s^{t}, S^{t}, \lambda^{t}\right)\right\}$ of energies is bounded, whence the desired result follows from Theorem 3.1 .

\subsubsection{Example: One-bar truss with a cable}

The example in this section is given to show a simple concrete mechanical structure covered by the general mathematical setting, and, moreover, to illustrate that the closedness of the feasible set is intimately connected with the boundedness of the energy (cf. Corollary 3.1). The closedness property will apparently be of paramount importance in order to establish the existence of optimal designs.

The example, shown in Section 1 in Figure 1, is a one-dimensional structure that consists of a bar, suspended with one cable. The initial slack is zero, and both lengths, specific weights and elastic modulii are one. The material volume for the bar and cable is $x$ and $X$, respectively. (The example will be reconsidered in Section 4.2, when maximal limits $\bar{\sigma}_{1}, \bar{\sigma}_{2}$ of stresses will be used, hence these additional symbols in the figure.)

If the load $f=1$, then the equilibrium relations in terms of displacement $u$, cable elongation $e$ and bar and cable force $(s, S)$ become

$$
u-e \leq 0, \quad S \geq 0, \quad(u-e) S=0, \quad S=X e, \quad-s=x u=1-S .
$$


It is always implicitly understood that the design variables are nonnegative. From (18) it is straightforward to verify that

$$
(s, S) \text { solves }(\mathcal{C})_{(x, X)}
$$

is equivalent to

$$
s=-\frac{x}{x+X}, \quad S=\frac{X}{x+X}, \quad x+X>0 .
$$

Hence the graph of the equilibrium mapping in terms of forces becomes

$$
\operatorname{gr} \mathcal{S}=\{(x, X, s, S) \mid S=X /(x+X), s=S-1, x+X>0\} .
$$

We now consider a structural optimization problem. Typically the upper-level feasible region is of the form

$$
Z_{\varepsilon}=\{(x, X, s, S) \mid(\varepsilon, \varepsilon) \leq(x, X) \leq(U, U)\},
$$

for some values $\varepsilon \geq 0$ and $U>\varepsilon$. To illustrate the effects of topological changes on the existence of optimal solutions, we here choose the design objective function to be to maximize the (unknown) displacement $u(x, X, s, S)$. Then, we can write the structural optimization problem as

$$
\left\{\begin{array}{cl}
\max _{(x, X, s, S)} & u(x, X, s, S), \\
\text { s.t. } & (x, X, s, S) \in Z_{\varepsilon} \cap \operatorname{gr} \mathcal{S} .
\end{array}\right.
$$

Consider first the sizing case, $\varepsilon>0$. It follows from (18) that $u(x, X)=1 /(x+X)$. It is therefore immediate to see that the optimal design is $x^{*}=X^{*}=\varepsilon$ and the optimal displacement $u^{*}=1 /(2 \varepsilon)$.

Consider next the topology case, $\varepsilon=0$. As opposed to the sizing case, the problem now lacks optimal solutions. Define for $n=1,2, \ldots$ the sequence

$$
\left(x_{n}, X_{n}, s_{n}, S_{n}\right)=\left(1 / n^{2}, 1 / n,-1 /(n+1), n /(n+1)\right) .
$$

It holds that, for all $n$ large enough, $\left(x_{n}, X_{n}, s_{n}, S_{n}\right)$ belongs to the feasible set $\mathcal{F}:=Z_{0} \cap$ gr $\mathcal{S}$ of the structural optimization problem, and

$$
\lim _{n \rightarrow \infty}\left(x_{n}, X_{n}, s_{n}, S_{n}\right)=(0,0,0,1) .
$$

However, clearly $(0,0,0,1) \notin$ gr $\mathcal{S}$, that is, the graph is not closed, and $(0,0,0,1)$ is infeasible! Moreover,

$$
u\left(x_{n}, X_{n}, s_{n}, S_{n}\right)=1 /\left(x_{n}+X_{n}\right)=n^{2} /(n+1) \rightarrow \infty,
$$

and therefore the structural optimization problem cannot possess any optimal solutions. Assuming that both design variables are nonzero, the energy is given by

$$
\mathcal{E}(x, X, s, S)=\frac{s^{2}}{2 x}+\frac{S^{2}}{2 X},
$$

and therefore

$$
\mathcal{E}\left(x_{n}, X_{n}, s_{n}, S_{n}\right)=\frac{n^{2}}{2(1+n)^{2}}+\frac{n^{3}}{2(1+n)^{2}}=\frac{n^{2}}{2(1+n)} \rightarrow \infty,
$$


that is, maximizing the displacement requires an unbounded elastic energy.

One could argue that maximizing the displacement is an objective that has no engineering meaning - it is rather the opposite that might be interesting. Assuming now that for problem statements that make engineering sense, the constraints and objective function in combination are such that an optimizing sequence does not demand unbounded energies, we again consider the set $L_{\nu}^{\mathcal{E}}$ defined in (17), where the value of $\nu$ is not important, as long as it is finite. (Later, for the minimum compliance problem, we will see that compliance equals energy, and since this quantity is to be minimized it is certainly finite. Moreover, in stress-constrained problems these constraints imply bounded energies.) Using (19) in (20) one shows that $\mathcal{E}(x, X, s, S) \leq \nu$ implies $x+X \geq 1 /(2 \nu)$, and therefore the constraint $x+X>0$ in $\operatorname{gr} \mathcal{S}$ is redundant. Consequently,

$$
\operatorname{gr} \mathcal{S} \cap L_{\nu}^{\mathcal{E}}=\{(x, X, s, S) \mid S=X /(x+X), s=S-1, x+X \geq 1 /(2 \nu)\},
$$

which is a closed set (as predicted by Corollary 3.1 )!

One main reason for working with forces as state variables is that the energy (for bounded design variables) is coercive with respect to forces; hence, if the energy is bounded, then the forces are bounded too. Indeed, if $(x, X, s, S)$ belongs to the new feasible set $\widetilde{\mathcal{F}}:=Z_{0} \cap \operatorname{gr} \mathcal{S} \cap L_{\nu}^{\mathcal{E}}$, then

$$
\frac{1}{2 C}\left(\|s\|^{2}+\|S\|^{2}\right) \leq \mathcal{E}(x, X, s, S) \leq \nu .
$$

This coercivity property does generally not hold in topology optimization when displacements are chosen as state variables, and therefore the displacements are then not bounded in general.

The reason for introducing the set $L_{\nu}^{\mathcal{E}}$ is twofold: first, in combination with the set $Z_{0}$ it bounds all the variables; second, when intersected with the set $\operatorname{gr} \mathcal{S}$ it produces a closed set. Therefore, whence $Z_{0}$ is also closed, it follows that the feasible set $\widetilde{\mathcal{F}}$ is compact, and therefore the topology optimization problem possesses optimal solutions for any proper and lsc objective function whose effective domain intersects $\widetilde{\mathcal{F}}$ (thanks to Weierstrass' Theorem).

\subsection{Design-to-displacement}

Although the equilibrium states $(s, S)$ are uniquely determined by the design $(x, X)$, the displacements $(u, e)$ are in general only unique when $(x, X)$ is strictly positive. Especially interesting then becomes the question to which, if any, displacement vector $(u, e)$ the sequence of equilibrium displacements $\left\{\left(u^{t}, e^{t}\right)\right\}$ converges when $\left\{\left(x^{t}, X^{t}\right)\right\}$ tends to a limit for which some elements are zero. To determine the answer to that question, we shall look at a particular kind of design sequence, in which small positive quantities are added to each element.

To that end, let $(\Psi, \Gamma)>(0,0)$ be arbitrary in $\mathbb{R}^{m} \times \mathbb{R}^{r_{2}}$. Then, $K(\Psi)$ and $k(\Gamma)$ are positive definite, and we can define an energy inner product in $\mathbb{R}^{m} \times \mathbb{R}^{r_{2}}$ as

$$
\left\langle\left(v_{1}, v_{2}\right),\left(w_{1}, w_{2}\right)\right\rangle:=v_{1}^{T} K(\Psi) w_{1}+v_{2}^{T} k(\Gamma) w_{2},
$$

and a corresponding energy norm as

$$
\left\|\left(v_{1}, v_{2}\right)\right\|_{\Psi, \Gamma}:=\sqrt{\left\langle\left(v_{1}, v_{2}\right),\left(v_{1}, v_{2}\right)\right\rangle}=\sqrt{v_{1}^{T} K(\Psi) v_{1}+v_{2}^{T} k(\Gamma) v_{2}} .
$$

Let $(x, X) \geq(0,0)$ be an arbitrary design and let $\mathcal{U}(x, X)$ denote the solution set of the problem $(\mathcal{P})_{(x, X)}$. One way to pick a unique displacement vector is to choose the one with least-energy norm. If $\mathcal{U}(x, X)$ is nonempty, then there indeed exists a unique such element,

$$
\{(\bar{u}(x, X), \bar{e}(x, X))\}:=\arg \min _{(u, e) \in \mathcal{U}(x, X)}\|(u, e)\|_{\Psi, \Gamma},
$$

since $\|\cdot\|_{\Psi, \Gamma}^{2}$ is strictly convex and $\mathcal{U}(x, X)$ is polyhedral convex. We will now establish that the sequence of displacements does converge to the least-energy displacement solution. 
Theorem 3.2 (Convergence to least-energy displacements). Let $(x, X) \geq(0,0)$ be a design for which $(\mathcal{P})_{(x, X)}$ has an optimal solution. Let $(\Psi, \Gamma)>(0,0)$ be arbitrary in $\mathbb{R}^{m} \times \mathbb{R}^{r_{2}}$, and set, for $\varepsilon>0$,

$$
\left(x_{\varepsilon}, X_{\varepsilon}\right):=(x, X)+\varepsilon(\Psi, \Gamma) .
$$

Further, denote by $\left(u_{\varepsilon}, e_{\varepsilon}\right)$ the unique optimal solution to the perturbed problem $(\mathcal{P})_{\left(x_{\varepsilon}, X_{\varepsilon}\right)}$. Then,

$$
\lim _{\varepsilon \rightarrow 0}\left(u_{\varepsilon}, e_{\varepsilon}\right)=(\bar{u}(x, X), \bar{e}(x, X))
$$

Proof. Let $(u, e)$ and $\left(u_{\varepsilon}, e_{\varepsilon}\right)$ be optimal in $(\mathcal{P})_{(x, X)}$ and $(\mathcal{P})_{\left(x_{\varepsilon}, X_{\varepsilon}\right)}$, respectively. Then, the vector $(u, e)$ [respectively, $\left.\left(u_{\varepsilon}, e_{\varepsilon}\right)\right]$, is feasible in the problem $(\mathcal{P})_{\left(x_{\varepsilon}, X_{\varepsilon}\right)}$ [respectively, $\left.(\mathcal{P})_{(x, X)}\right]$, so it follows that

$$
\frac{1}{2} u^{T} K(x) u+\frac{1}{2} e^{T} k(X) e-f^{T} u \leq \frac{1}{2} u_{\varepsilon}^{T} K(x) u_{\varepsilon}+\frac{1}{2} e_{\varepsilon}^{T} k(X) e_{\varepsilon}-f^{T} u_{\varepsilon}
$$

and

$$
\frac{1}{2} u_{\varepsilon}^{T} K\left(x_{\varepsilon}\right) u_{\varepsilon}+\frac{1}{2} e_{\varepsilon}^{T} k\left(X_{\varepsilon}\right) e_{\varepsilon}-f^{T} u_{\varepsilon} \leq \frac{1}{2} u^{T} K\left(x_{\varepsilon}\right) u+\frac{1}{2} e^{T} k\left(X_{\varepsilon}\right) e-f^{T} u .
$$

Adding (22) and (23) yields that

$$
\frac{\varepsilon}{2}\left[u_{\varepsilon}^{T} K(\Psi) u_{\varepsilon}+e_{\varepsilon}^{T} k(\Gamma) e_{\varepsilon}\right] \leq \frac{\varepsilon}{2}\left[u^{T} K(\Psi) u+e^{T} k(\Gamma) e\right],
$$

that is,

$$
\left\|\left(u_{\varepsilon}, e_{\varepsilon}\right)\right\|_{\Psi, \Gamma} \leq\|(u, e)\|_{\Psi, \Gamma} .
$$

Clearly, then, the sequence $\left\{\left(u_{\varepsilon}, e_{\varepsilon}\right)\right\}$ is bounded, and since the pair $(u, e)$ was arbitrary in $\mathcal{U}(x, X)$, each of the limit points $(\bar{u}, \bar{e})$ of the sequence $\left\{\left(u_{\varepsilon}, e_{\varepsilon}\right)\right\}$ satisfies, in particular, the relation

$$
\|(\bar{u}, \bar{e})\|_{\Psi, \Gamma} \leq\|(\bar{u}(x, X), \bar{e}(x, X))\|_{\Psi, \Gamma},
$$

where $(\bar{u}(x, X), \bar{e}(x, X))$ was defined in (21). But the least-energy displacement is unique, so the limit point must be unique, and $(\bar{u}, \bar{e})=(\bar{u}(x, X), \bar{e}(x, X))$ must hold, whence the result follows.

In principle, this result on the convergence to least-energy displacements can be obtained from more general principles for regularizations of ill-posed variational inequalities ([Bro66]) and perturbations of variational inequalities ([Sta69]). However, we believe it is more instructive and convenient for the reader with our direct proof rather than specializing the general frameworks to our notation.

\subsection{Design-to-overall state}

In this section, we establish the convergence rate and local Lipschitz continuity of the sequences $\left\{\left(u_{\varepsilon}, e_{\varepsilon}\right)\right\},\left\{\left(s_{\varepsilon}, S_{\varepsilon}\right)\right\}$ and $\left\{\lambda_{\varepsilon}\right\}$ simultaneously. These results hinge on the use of error bounds for the solutions to affine variational inequality problems, first established by Luo and Tseng [LuT92, LuT97] (see also [LPR96, Theorem 2.3.3 and 2.3.5]). Letting SOL $(q, Q, Y)$ denote the solution set of the $\operatorname{AVI}(q, Q, Y)$ [see (14)], dist $[z, Z]$ the least Euclidean distance from a vector $z$ to a set $Z$, and $\operatorname{proj}[z, Z]$ the Euclidean projection of a vector $z$ onto a set $Z$, we then have the following: if $\operatorname{SOL}(q, Q, Y)$ is nonempty, then there exist positive constants $\tau$ and $\delta$ such that

$$
\operatorname{dist}[y, \operatorname{SOL}(q, Q, Y)] \leq \tau\|y-\operatorname{proj}[(y-Q y-q), Y]\|
$$

holds for all $y \in \mathbb{R}^{n}$ with $\|y-\operatorname{proj}[(y-Q y-q), Y]\| \leq \delta$. 
Theorem 3.3 (Convergence rate of forces and displacements). Let $(x, X) \geq(0,0)$ be a design for which $(\mathcal{P})_{(x, X)}$ has an optimal solution, and $(s, S, \lambda)$ be the optimal solution to $(\mathcal{C})_{(x, X)}$. Let $(\Psi, \Gamma)>(0,0)$ be arbitrary in $\mathbb{R}^{m} \times \mathbb{R}^{r_{2}}$, and set, for $\varepsilon>0$,

$$
\left(x_{\varepsilon}, X_{\varepsilon}\right):=(x, X)+\varepsilon(\Psi, \Gamma) .
$$

Further, denote by $\left(u_{\varepsilon}, e_{\varepsilon}\right)$ the optimal solution to the perturbed problem $(\mathcal{P})_{\left(x_{\varepsilon}, X_{\varepsilon}\right)}$, and by $\left(s_{\varepsilon}, S_{\varepsilon}, \lambda_{\varepsilon}\right)$ the corresponding optimal solution to $(\mathcal{C})_{\left(x_{\varepsilon}, X_{\varepsilon}\right)}$. Then, for some positive constant $\hat{\tau}$,

$$
\operatorname{dist}\left[\left(u_{\varepsilon}, e_{\varepsilon}\right), \mathcal{U}(x, X)\right] \leq \hat{\tau} \varepsilon \quad \text { and } \quad\left\|\left(s_{\varepsilon}, S_{\varepsilon}, \lambda_{\varepsilon}\right)-(s, S, \lambda)\right\| \leq \hat{\tau} \varepsilon
$$

holds for all sufficiently small $\varepsilon>0$.

Proof. We begin by stating the equilibrium conditions for forces and displacements of the perturbed problem as an AVI of the form AVI $\left(q_{\varepsilon}, Q_{\varepsilon}, Y\right)$. Let

$$
y_{\varepsilon}:=\left(\begin{array}{c}
u_{\varepsilon} \\
e_{\varepsilon} \\
s_{\varepsilon} \\
S_{\varepsilon} \\
\lambda_{\varepsilon}
\end{array}\right), \quad Q_{\varepsilon}:=Q+\varepsilon\left(\begin{array}{ccccc}
0 & 0 & 0 & 0 & 0 \\
D(\Psi) B & 0 & 0 & 0 & 0 \\
0 & k(\Gamma) & 0 & 0 & 0 \\
0 & 0 & 0 & 0 & 0 \\
0 & 0 & 0 & 0 & 0
\end{array}\right), \quad \text { and } \quad q_{\varepsilon}=q .
$$

We then obtain from the above error bound that

$$
\begin{aligned}
& \operatorname{dist}\left[y_{\varepsilon}, \operatorname{SOL}(q, Q, Y)\right] \leq \tau\left\|y_{\varepsilon}-\operatorname{proj}\left[\left(y_{\varepsilon}-Q y_{\varepsilon}-q\right), Y\right]\right\| \\
&=\tau\left\|\left(\begin{array}{c}
u_{\varepsilon} \\
e_{\varepsilon} \\
s_{\varepsilon} \\
S_{\varepsilon} \\
\lambda_{\varepsilon}
\end{array}\right)-\left(\begin{array}{c}
u_{\varepsilon} \\
e_{\varepsilon}+\varepsilon D(\Psi) B u_{\varepsilon} \\
s_{\varepsilon}+\varepsilon k(\Gamma) e_{\varepsilon} \\
{\left[S_{\varepsilon}+C_{2} u_{\varepsilon}-e_{\varepsilon}-g_{2}\right]_{+}} \\
{\left[\lambda_{\varepsilon}+C_{1} u_{\varepsilon}-g_{1}\right]_{+}}
\end{array}\right)\right\| \\
&=\tau \varepsilon\left\|\left(\begin{array}{c}
0 \\
D(\Psi) B u_{\varepsilon} \\
k(\Gamma) e_{\varepsilon} \\
0 \\
0
\end{array}\right)\right\| \leq \hat{\tau} \varepsilon,
\end{aligned}
$$

where $[y]_{+}:=\max \{0, y\}$, taken component-wise, and the second inequality holds because the sequence $\left\{\left(u_{\varepsilon}, e_{\varepsilon}\right)\right\}$ is bounded (cf. Theorem 3.2). The result follows.

The error bound (24) is clearly local, since it is valid only near the solution set $\operatorname{SOL}(q, Q, Y)$. A global version where (24) is valid for any $y$ (and in fact for any fixed vector $q$ ) however holds under the condition that the problem $\operatorname{AVI}(0, Q, Y)$ has zero as the unique solution (cf. [LuT97]). Using this result and a similar proof technique to that which is used in the above result, we next establish that the equilibrium state $(u, e, s, S, \lambda)$ varies in a locally Lipschitz continuous manner with the design $(x, X)$ in the positive orthant.

Theorem 3.4 (Local Lipschitz continuity of the equilibrium state). Let $D \subset \mathbb{R}_{++}^{m} \times \mathbb{R}_{++}^{r_{2}}$ be a nonempty, convex and compact set. Let $\left(x^{1}, X^{1}\right)$ and $\left(x^{2}, X^{2}\right)$ be two arbitrary designs in $D$. Denote the respective equilibrium states by $y^{1}:=\left(u^{1}, e^{1}, s^{1}, S^{1}, \lambda^{1}\right)$ and $y^{2}:=\left(u^{2}, e^{2}, s^{2}, S^{2}, \lambda^{2}\right)$. Then, for some nonnegative constant $\kappa$ (depending on $D$ ),

$$
\left\|y^{2}-y^{1}\right\| \leq \kappa\left\|\left(x^{2}, X^{2}\right)-\left(x^{1}, X^{1}\right)\right\| .
$$


Proof. Consider the AVI $\left(0, Q\left(x^{1}, X^{1}\right), Y\right)$. Since $\left(x^{1}, X^{1}\right)>(0,0)$, the solution to this problem is unique. Clearly, the zero solution is one solution to the problem, whence it is also the unique solution. Therefore, according to the above, the error bound (24) is valid globally for the $\operatorname{AVI}\left(q, Q\left(x^{1}, X^{1}\right), Y\right)$. Applying this error bound to the $\operatorname{AVI}\left(q, Q\left(x^{1}, X^{1}\right), Y\right)$ with $y:=y^{2}$, we obtain for some $\tau, \bar{\tau}>0$, that

$$
\begin{aligned}
\left\|y^{2}-y^{1}\right\| & \leq \tau\left\|\left(0, D\left(x^{2}-x^{1}\right) B u^{2}, k\left(X^{2}-X^{1}\right) e^{2}, 0,0\right)\right\| \\
& \leq \bar{\tau}\left\|\left(x^{2}, X^{2}\right)-\left(x^{1}, X^{1}\right)\right\| \cdot\left\|\left(u^{2}, e^{2}\right)\right\| .
\end{aligned}
$$

Since the equilibrium state is bounded over $D$, the result (25) follows, with $\kappa$ equal to $\bar{\tau}$ times the supremum of the length of the $(u, e)$ component over the equilibrium states in $D$.

This result extends that of Christiansen et al. [CPW01] for the cable-less case, where local Lipschitz continuity is established for $y$ by the use of Robinson's [Rob80, Rob91] sensitivity analysis of parametric variational inequality problems.

\section{Cable suspended trusses}

\subsection{Minimum compliance}

\subsubsection{The design optimization model}

In case of unilateral constraints due to elastic cables, we define the (extended) compliance as

$$
\frac{1}{2}\left(f^{T} u+g_{2}^{T} S\right)
$$

assuming $S_{j}=0$ for all $j \notin \mathcal{J}(X)$. (Note that $C_{1}, \lambda$, and $g_{1}$ do not enter the problem.) Minimizing this objective hence means to minimize displacements weighted by forces plus cable forces weighted by slacks. This choice of design (or, upper level) objective function actually coincides with the objective function in $(\mathcal{C})_{(x, X)}$, which we now turn to establish.

Using (4) and (12) in the objective function appearing in the principle of minimum of complementary energy, this objective becomes

$$
\begin{aligned}
\mathcal{E}_{\mathcal{I}, \mathcal{J}}(x, X, s, S) & :=\frac{1}{2} \sum_{i \in \mathcal{I}(x)} \frac{s_{i}^{2}}{E x_{i}}+\sum_{j \in \mathcal{J}(X)}\left(\frac{\left(L_{j} S_{j}\right)^{2}}{2 E_{c} X_{j}}+\left(g_{2}\right)_{j} S_{j}\right) \\
& =\frac{1}{2} u^{T} \sum_{i \in \mathcal{I}(x)} B_{i}^{T} s_{i}+\sum_{j \in \mathcal{J}(X)}\left(\frac{1}{2} S_{j} e_{j}+\left(g_{2}\right)_{j} S_{j}\right) .
\end{aligned}
$$

The complementarity part of (10) gives $S_{j} e_{j}=\gamma_{j}^{T} u S_{j}-\left(g_{2}\right)_{j} S_{j}$, which simplifies the expression further to

$$
\frac{1}{2} u^{T}\left(\sum_{i \in \mathcal{I}(x)} B_{i}^{T} s_{i}+\sum_{j \in \mathcal{J}(X)} S_{j} \gamma_{j}\right)+\frac{1}{2} \sum_{j \in \mathcal{J}(X)}\left(g_{2}\right)_{j} S_{j},
$$

which, by (13), reduces to

$$
\frac{1}{2}\left(f^{T} u+\sum_{j \in \mathcal{J}(X)}\left(g_{2}\right)_{j} S_{j}\right),
$$


and therefore

$$
\mathcal{E}_{\mathcal{I}, \mathcal{J}}(x, X, s, S)=\frac{1}{2}\left(f^{T} u+g_{2}^{T} S\right)
$$

on $\operatorname{gr} \mathcal{S}$.

We let the $(x, X)$ be design variables, having bounds

$$
0 \leq \underline{x} \leq x \leq \bar{x} ; \quad 0 \leq \underline{X} \leq X \leq \bar{X} .
$$

With $\underline{x}=0$ and $\underline{X}=0$ one obtains true topology optimization in a framework that looks like a sizing problem.

The design problem of minimizing the compliance, given a limited amount of cable and structure material, can now be posed as

$$
\left\{\begin{aligned}
\min _{(x, X, s, S)} & \mathcal{E}(x, X, s, S), \\
\text { s.t. } & \left\{\begin{array}{l}
\underline{x} \leq x \leq \bar{x}, \\
\underline{X} \leq X \leq \bar{X}, \quad 1_{m}^{T} x \leq v, \\
(s, S) \text { solves }(\mathcal{C})_{(x, X)} .
\end{array}\right.
\end{aligned}\right.
$$

\subsubsection{Example: Crane subject to stochastic wind force}

Suppose that the external loads $f=f_{\omega}$, where $\omega$ belongs to a probability space $(\Omega, \mathcal{A}, P)$. Here, $\Omega$ is the sample space, $p$ the probability density function, and $P$ the cumulative distribution function.

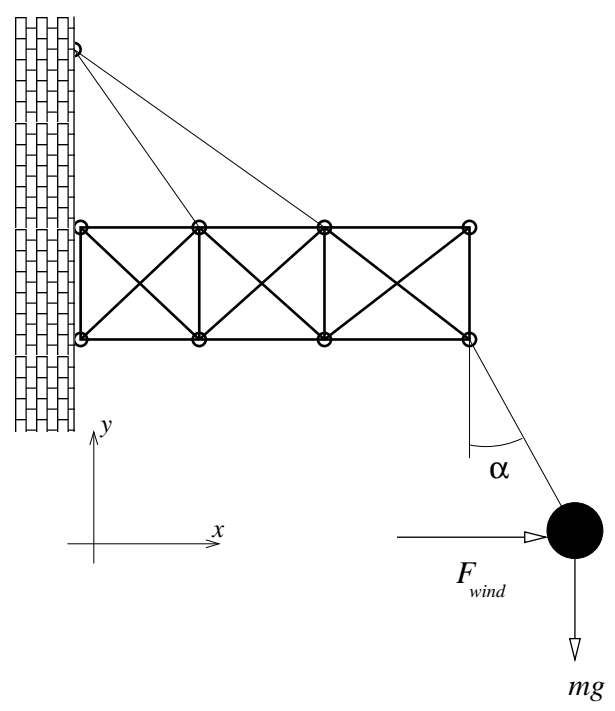

Figure 3: A crane which carries a load subjected to a random wind force.

Consider the cable-suspended crane in Figure 3. We suppose that the initial slacks are zero and that there are no contact constraints. Then the compliance can be expressed in terms of displacements as

$$
\bar{f}(u):=\frac{1}{2} f^{T} u .
$$


Assume now that the crane carries a weight $m g$ and that a wind force on the weight acts horizontally with a random magnitude. The wind force is assumed to take values according to the probability density function shown in Figure 4. Hence the load vector $f \in \mathbb{R}^{12}$ depends on the value $\omega=F_{\text {wind }}$ :

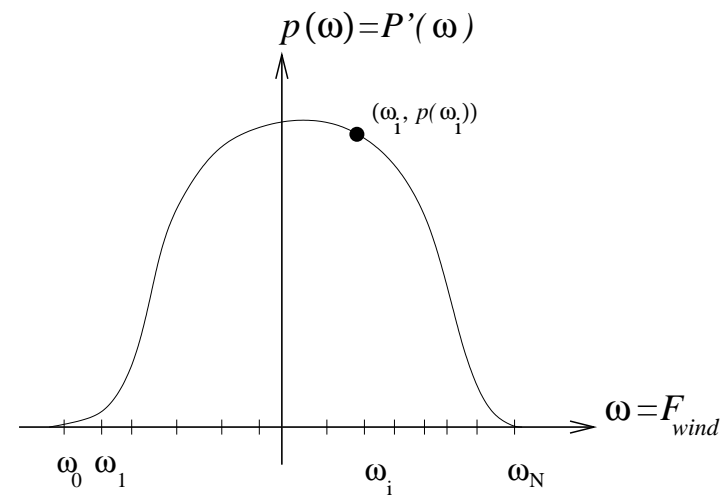

Figure 4: Probability density function for the wind force.

$$
f_{\omega}=\left(\begin{array}{c}
0 \\
\vdots \\
0 \\
\omega \\
-m g \\
0 \\
\vdots \\
0
\end{array}\right), \quad \omega \in \Omega=[\underline{\omega}, \bar{\omega}]=\left[\omega_{0}, \omega_{N}\right]
$$

Since the load vector depends on $\omega$, so does $(\mathcal{P})_{(x, X)}$, and we write $(\mathcal{P})_{(x, X)}(\omega)$ for the equilibrium problem and $[u(\omega), e(\omega)]$ for its solution. Then the compliance can be written as $\bar{f}_{\omega}(u(\omega)):=$ $\frac{1}{2} f_{\omega}^{T} u(\omega)$. This value is different for different values of the stochastic variable $\omega$, but we need a single value in the objective of the design optimization problem. One natural choice is to take the expected value of the compliance as the objective. The minimum compliance problem can for this example then be formulated as

$$
\begin{cases}\min _{(x, X, u, e)} & E_{\omega}\left[\bar{f}_{\omega}(u(\omega))\right], \\
\text { s.t. } & \left\{\begin{array}{l}
\underline{x} \leq x \leq \bar{x}, \quad 1_{m}^{T} x \leq v, \\
\underline{X} \leq X \leq \bar{X}, \quad 1_{r_{2}}^{T} X \leq V, \\
u(\omega), e(\omega) \text { solves }(\mathcal{P})_{(x, X)}(\omega), \quad \omega \in \Omega .
\end{array}\right.\end{cases}
$$

The expected value is given by

$$
E_{\omega}\left[\bar{f}_{\omega}(u(\omega))\right]=\frac{1}{2} \int_{\underline{\omega}}^{\bar{\omega}} f_{\omega}^{T} u(\omega) p(\omega) d \omega .
$$

For each design candidate $(x, X)$ it is in general impossible to calculate the structure's displacement response $u(\omega)$ for every $\omega \in \Omega$, unless $\Omega$ is a discrete sample space. Hence, when $\Omega$ is continuous as 
in this example, one way is to discretize $\Omega$, see Figure 4. Applying Simpsons's rule on the integral in (28), we can approximate the expected value as

$$
E_{\omega}\left[\bar{f}_{\omega}(u(\omega))\right] \approx \frac{1}{2} \sum_{i=1}^{N-1} f_{\omega_{i}}^{T} u\left(\omega_{i}\right) p\left(\omega_{i}\right) \frac{h_{i}+h_{i+1}}{2}=\frac{1}{2} \sum_{\ell \in \mathcal{L}} \rho_{\ell}\left(f^{\ell}\right)^{T} u^{\ell},
$$

where

$$
f^{\ell}=f_{\omega_{\ell}}, \quad u^{\ell}=u\left(\omega_{\ell}\right), \quad \rho_{\ell}=p\left(\omega_{\ell}\right) \frac{h_{\ell}+h_{\ell+1}}{2}, \quad \mathcal{L}=\{1, \ldots, N-1\},
$$

and

$$
h_{\ell}=\omega_{\ell}-\omega_{\ell-1}, \quad \ell=1, \ldots, N .
$$

Before continuing with the problem formulation, we introduce the new notation $(\tilde{u}, \tilde{e}, \tilde{s}, \tilde{S})$ to denote the collection of vectors $\left(u^{\ell}, e^{\ell}, s^{\ell}, S^{\ell}\right)_{\ell \in \mathcal{L} \text {. }}$.

Consequently, we arrive at the problem formulation

$$
\left\{\begin{aligned}
\min _{(x, X, \tilde{u}, \tilde{e})} & \frac{1}{2} \sum_{\ell \in \mathcal{L}} \rho_{\ell}\left(f^{\ell}\right)^{T} u^{\ell}, \\
\text { s.t. } & \left\{\begin{array}{l}
\underline{x} \leq x \leq \bar{x}, \quad 1_{m}^{T} x \leq v, \\
\underline{X} \leq X \leq \bar{X}, \quad 1_{r_{2}}^{T} X \leq V, \\
\left(u^{\ell}, e^{\ell}\right) \text { solves }(\mathcal{P})_{(x, X)}\left(\omega_{\ell}\right), \quad \ell \in \mathcal{L} .
\end{array}\right.
\end{aligned}\right.
$$

This looks like a traditional multiple load-case formulation. Here, however, the weight $\rho_{\ell}$ (henceforth presumed strictly positive for every $\ell \in \mathcal{L}$ ) in the objective function for each load-case is determined by a probability function for some event in the structure's environment.

We can now write down the general stochastic minimum compliance problem:

$$
\left(\mathcal{P}_{1}\right) \begin{cases}\min _{(x, X, \tilde{s}, \tilde{S})} & c^{f}(x, X, \tilde{s}, \tilde{S}):=\sum_{\ell \in \mathcal{L}} \rho_{\ell} \mathcal{E}^{\ell}\left(x, X, s^{\ell}, S^{\ell}\right)= \\
& \sum_{\ell \in \mathcal{L}} \rho_{\ell}\left[\frac{1}{2} \sum_{i \in \mathcal{I}(x)} \frac{\left(s_{i}^{\ell}\right)^{2}}{E x_{i}}+\sum_{j \in \mathcal{J}(X)}\left(\frac{\left(L_{j} S_{j}^{\ell}\right)^{2}}{2 E_{c} X_{j}}+\left(g_{2}\right)_{j} S_{j}^{\ell}\right)\right], \\
\text { s.t. } \quad\left\{\begin{array}{l}
\underline{x} \leq x \leq \bar{x}, \quad 1_{m}^{T} x \leq v, \\
\underline{X} \leq X \leq \bar{X}, \quad 1_{r_{2}}^{T} X \leq V, \\
\left(s^{\ell}, S^{\ell}\right) \text { solves }(\mathcal{C})_{(x, X)}\left(\omega_{\ell}\right), \quad \ell \in \mathcal{L} .
\end{array}\right.\end{cases}
$$

\subsubsection{Existence of optimal designs}

The following result establishes the existence of optimal solutions to this problem. (We note that in the statement of the result, the existence of a feasible solution is guaranteed whenever the bounds on the design $(x, X)$ are such that a strictly positive design is feasible, which, clearly, will always be the case.)

Theorem 4.1 (Existence of optimal solutions to $\left(\mathcal{P}_{1}\right)$ ). Suppose the feasible set $\mathcal{F}^{\mathcal{P}_{1}}$ of $\left(\mathcal{P}_{1}\right)$ is nonempty. Then, there exists at least one optimal solution to $\left(\mathcal{P}_{1}\right)$. 
Proof. The design objective is to minimize a (strictly positive) weighted sum of terms with $\left(s^{\ell}, S^{\ell}\right)$ being the optimal solution to $(\mathcal{C})_{(x, X)}\left(\omega_{\ell}\right)$. Hence, by the feasibility assumption (which implies that the functions $\mathcal{E}^{\ell}$ are proper with respect to $\mathcal{F}^{\mathcal{P}_{1}}$ ), without any loss of generality, we may assume that all feasible solutions satisfy

$$
\mathcal{E}^{\ell}\left(x, X, s^{\ell}, S^{\ell}\right) \leq \nu_{\ell}<\infty, \quad \ell \in \mathcal{L} .
$$

We may therefore replace the constraints of $\left(\mathcal{P}_{1}\right)$ with the design constraints and the constraints that

$$
\left(x, X, s^{\ell}, S^{\ell}\right) \in \operatorname{gr} \mathcal{S}_{\ell} \cap L_{\nu_{\ell}}^{\mathcal{E}^{\ell}}, \quad \ell \in \mathcal{L},
$$

which forms a closed set by Corollary 3.1. Hence, the feasible set of $\left(\mathcal{P}_{1}\right)$ is closed as well as nonempty. As remarked above, the upper-level objective function is proper with respect to $\mathcal{F}^{\mathcal{P}_{1}}$, and it is further lsc (cf. Lemma 3.2) and coercive, since it is coercive in $(\tilde{s}, \tilde{S})$ and the feasible set in terms of $(x, X)$ is bounded. Hence, Weierstrass' Theorem applies.

\subsubsection{Convex-concave saddle-point and convex programming formulations}

When the (extended) compliance is used as the upper-level design objective, the optimal design problem can be equivalently rewritten as a convex-concave saddle-point problem, or a convex (but nondifferentiable) optimization problem. This has an immediate advantage computationally, since it means that the problem can be attacked by techniques from convex programming, but it can also be utilized as an alternative formulation when establishing, for example, the existence of optimal solutions. In the case of the current problem $\left(\mathcal{P}_{1}\right)$, the equivalent saddle-point formulation has the following form:

$$
\left(\mathcal{S P}_{1}\right)\left\{\begin{array}{l}
\text { find }\left(x^{*}, X^{*}, u^{*}, e^{*}\right) \in \mathcal{Z} \times \mathcal{U}: \\
\mathcal{J}\left(x, X, u^{*}, e^{*}\right) \leq \mathcal{J}\left(x^{*}, X^{*}, u^{*}, e^{*}\right) \leq \mathcal{J}\left(x^{*}, X^{*}, u, e\right), \quad \forall(x, X, u, e) \in \mathcal{Z} \times \mathcal{U},
\end{array}\right.
$$

where

$$
\begin{aligned}
\mathcal{J}(x, X, u, e) & :=\sum_{\ell \in \mathcal{L}} \rho_{\ell}\left(\frac{1}{2} \sum_{i=1}^{m} x_{i}\left(u^{\ell}\right)^{T} B_{i}^{T} E B_{i} u^{\ell}+\frac{1}{2} \sum_{j=1}^{r_{2}} \frac{E_{c} X_{j}}{L_{j}^{2}}\left(e_{j}^{\ell}\right)^{2}-\left(f^{\ell}\right)^{T} u^{\ell}\right), \\
\mathcal{Z} & :=\left\{(x, X) \mid(\underline{x}, \underline{X}) \leq(x, X) \leq(\bar{x}, \bar{X}) ; 1_{m}^{T} x \leq v ; 1_{r_{2}}^{T} X \leq V\right\}, \\
\mathcal{U} & :=\left\{(u, e)=\left(u^{\ell}, e^{\ell}\right)_{\ell \in \mathcal{L}} \mid \gamma_{j}^{T} u^{\ell}-e_{j}^{\ell} \leq\left(g_{2}\right)_{j}, \quad j=1, \ldots, r_{2}, \quad \ell \in \mathcal{L}\right\} .
\end{aligned}
$$

A convex programming formulation in terms of only displacement variables can be obtained by eliminating the design variables from the problem:

$$
\left(\mathcal{C P}_{1}\right)\left\{\begin{array}{l}
\text { find }\left(u^{*}, e^{*}\right) \in \mathcal{U}: \\
\mathcal{J}^{*}\left(u^{*}, e^{*}\right) \leq \mathcal{J}^{*}(u, e), \quad \forall(u, e) \in \mathcal{U}
\end{array}\right.
$$

where

$$
\mathcal{J}^{*}(u, e):=\max _{(x, X) \in \mathcal{Z}} \mathcal{J}(x, X, u, e) .
$$


(The function $\mathcal{J}^{*}$ is a finite convex function.) The minimum (extended) compliance objective appears if we eliminate the displacement variables: it holds that

$$
\mathcal{J}_{*}(x, X):=\inf _{(u, e) \in \mathcal{U}} \mathcal{J}(x, X, u, e)=-\frac{1}{2} \sum_{\ell \in \mathcal{L}} \rho_{\ell}\left(f^{T} u^{\ell}+g_{2}^{T} S^{\ell}\right),
$$

where $S^{\ell}$ is the equilibrium tensile force solution of $(\mathcal{C})_{(x, X)}\left(\omega_{\ell}\right)$. A nested, convex optimization formulation of the design problem in terms of the design variables only is then obtained as:

$$
\left(\mathcal{C P}_{2}\right)\left\{\begin{array}{l}
\text { find }\left(x^{*}, X^{*}\right) \in \mathcal{Z}: \\
\mathcal{J}_{*}\left(x^{*}, X^{*}\right) \geq \mathcal{J}_{*}(x, X), \quad \forall(x, X) \in \mathcal{Z},
\end{array}\right.
$$

where we remark that $\mathcal{J}_{*}$ is a concave function.

Obviously, this development can be done also in the presence of unilateral contact conditions, for the problem $(\mathcal{P})$ in case the design objective is minimal extended compliance. For general references on saddle-point and convex programming formulations in truss topology optimization including unilateral constraints, we refer to [PeK94, PeP97].

We note, finally, that the existence of optimal solutions can be established also for more general upper-level design objectives, as has been done, for example, in [CPW01], in the case of cable-less structures. Essentially, they provide two types of existence results. The first is similar to Theorem 4.1, in that it relies on Weierstrass' Theorem, the main presumptions being the closedness of the set of feasible solutions and the coercivity of the upper-level objective function. The second result, which is close in spirit to the existence result in quadratic programming in [FrW56], amounts to replacing the coercivity assumption on the design objective with the less stringent set of assumptions that it is lower bounded on the graph of equilibrium solutions and quadratic in the lower-level variables, and further that a specially constructed lower level set is closed. The last presumption is equivalent to assuming that for all sufficiently good feasible designs (with respect to the design objective), the set of equilibrium displacements can be taken to lie in a compact set, a presumption which we have seen above to be a rather natural assumption to make.

\subsection{5 $\varepsilon$-perturbation}

In topology optimization, the lower design bounds $(\underline{x}, \underline{X})$ are taken to be zero. According to Theorem 4.1, this is, in principle, also legitimate from a solvability point of view. However, for designs with vanishing material, neither equilibrium states nor derivatives needed in a first-order method may be computable. Therefore, a common strategy is to replace the zero lower design bound with a small lower bound $\varepsilon>0$, thereby allowing for the computations needed in a standard nested approach.

When perturbing the problem by enforcing a lower bound $\varepsilon>0,(\mathcal{P})_{(x, X)}$ is always uniquely solvable, so we can switch from $(\mathcal{C})_{(x, X)}$ to $(\mathcal{P})_{(x, X)}$, as it is generally considered easier to work in the displacement space. The $\varepsilon$-perturbed problem reads

$$
\left(\mathcal{P}_{1}^{\varepsilon}\right)\left\{\begin{array}{l}
\min _{(x, X, \tilde{u}, \tilde{e})} c^{d}(x, X, \tilde{u}, \tilde{e}):=\frac{1}{2} \sum_{\ell \in \mathcal{L}} \rho_{\ell}\left(f^{\ell}\right)^{T} u^{\ell}+\frac{1}{2} \sum_{\ell \in \mathcal{L}} \rho_{\ell} \sum_{j=1}^{r_{2}} \frac{\left(X_{j} E_{c}\right)}{L_{j}^{2}} \cdot\left(g_{2}\right)_{j} e_{j}^{\ell}, \\
\text { s.t. }\left\{\begin{array}{l}
\varepsilon 1_{m} \leq x \leq \bar{x}, \quad 1_{m}^{T} x \leq v, \\
\varepsilon 1_{r_{2}} \leq X \leq \bar{X}, \quad 1_{r_{2}}^{T} X \leq V, \\
\left(u^{\ell}, e^{\ell}\right) \text { solves }(\mathcal{P})_{(x, X)}\left(\omega_{\ell}\right), \quad \ell \in \mathcal{L} .
\end{array}\right.
\end{array}\right.
$$

The reader should note that we use the notation $c^{f}$ and $c^{d}$, respectively, for the design objectives in the problems $\left(\mathcal{P}_{1}\right)$ and $\left(\mathcal{P}_{1}^{\varepsilon}\right)$, in order to distinguish the use of force and displacement 
variables in the equilibrium conditions. However, the two objectives are equal when evaluated at equilibrium points, which is of course always the case in the structural optimization problems, and therefore we can interchange them whenever desired. We shall also let the entire vector $(\tilde{u}, \tilde{e}, \tilde{s}, \tilde{S})$ [respectively, $\left(\tilde{u}_{\varepsilon}, \tilde{e}_{\varepsilon}, \tilde{s}_{\varepsilon}, \tilde{S}_{\varepsilon}\right)$ ] be part of the optimal solution to the problem $\left(\mathcal{P}_{1}\right)$ [respectively, $\left(\mathcal{P}_{1}^{\varepsilon}\right)$ ], although it is not part of the optimization formulation in its entirety. (It, however, of course constitutes the solution to the primal-dual pair of problems $(\mathcal{C})_{(x, X)}\left(\omega_{\ell}\right)$ and $(\mathcal{P})_{(x, X)}\left(\omega_{\ell}\right)$ [respectively, $(\mathcal{C})_{\left(x_{\varepsilon}, X_{\varepsilon}\right)}\left(\omega_{\ell}\right)$ and $\left.\left.(\mathcal{P})_{\left(x_{\varepsilon}, X_{\varepsilon}\right)}\left(\omega_{\ell}\right)\right].\right)$

The following result motivates the use of the above problem manipulation.

Theorem 4.2 (Convergence of $\varepsilon$-perturbed solutions). Suppose the feasible set $\mathcal{F}^{\mathcal{P}_{1}}$ of $\left(\mathcal{P}_{1}\right)$ is nonempty. For each $\varepsilon>0$, let $\left(x_{\varepsilon}^{*}, X_{\varepsilon}^{*}, \tilde{u}_{\varepsilon}^{*}, \tilde{e}_{\varepsilon}^{*}, \tilde{s}_{\varepsilon}^{*}, \tilde{S}_{\varepsilon}^{*}\right)$ denote an arbitrary optimal solution to $\left(\mathcal{P}_{1}^{\varepsilon}\right)$. Then, the sequence $\left\{\left(x_{\varepsilon}^{*}, X_{\varepsilon}^{*}, \tilde{s}_{\varepsilon}^{*}, \tilde{S}_{\varepsilon}^{*}\right)\right\}$ is bounded, and converges to the optimal solution set $\mathrm{SOL}\left(\mathcal{P}_{1}\right)$ of $\left(\mathcal{P}_{1}\right)$, in the sense that

$$
\left\{\min _{(x, X, \tilde{s}, \tilde{S}) \in \operatorname{SOL}\left(\mathcal{P}_{1}\right)}\left\|\left(x_{\varepsilon}^{*}, X_{\varepsilon}^{*}, \tilde{s}_{\varepsilon}^{*}, \tilde{S}_{\varepsilon}^{*}\right)-(x, X, \tilde{s}, \tilde{S})\right\|\right\} \rightarrow 0 .
$$

Moreover, $\left\{c^{f}\left(x_{\varepsilon}^{*}, X_{\varepsilon}^{*}, \tilde{s}_{\varepsilon}^{*}, \tilde{S}_{\varepsilon}^{*}\right)\right\}$ and $\left\{c^{d}\left(x_{\varepsilon}^{*}, X_{\varepsilon}^{*}, \tilde{u}_{\varepsilon}^{*}, \tilde{e}_{\varepsilon}^{*}\right)\right\}$ converges to the optimal value of $\left(\mathcal{P}_{1}\right)$.

Proof. According to Theorem 4.1, an optimal solution exists to the problem $\left(\mathcal{P}_{1}^{\varepsilon}\right)$ for every $\varepsilon>0$, as well as to the problem $\left(\mathcal{P}_{1}\right)$. Consider first the sequence $\left\{\left(x_{\varepsilon}^{*}, X_{\varepsilon}^{*}\right)\right\}$. Clearly, this sequence is bounded since the feasible sets of $\left(\mathcal{P}_{1}^{\varepsilon}\right)$ in $\left(x_{\varepsilon}, X_{\varepsilon}\right)$ are bounded, as well as that in $(x, X)$ of $\left(\mathcal{P}_{1}\right)$. We further note that since these sets increase with a decreasing $\varepsilon$, the sequence $\left\{c^{f}\left(x_{\varepsilon}^{*}, X_{\varepsilon}^{*}, \tilde{s}_{\varepsilon}^{*}, \tilde{S}_{\varepsilon}^{*}\right)\right\}$ is decreasing. In particular, it is then bounded from above. We may then use Theorem 3.1 to conclude that also the sequence $\left\{\left(\tilde{s}_{\varepsilon}^{*}, \tilde{S}_{\varepsilon}^{*}\right)\right\}$ is bounded and further that if $(\bar{x}, \bar{X})$ is an arbitrary limit point of the sequence $\left\{\left(x_{\varepsilon}^{*}, X_{\varepsilon}^{*}\right)\right\}$ then $\left\{\left(s_{\varepsilon}^{\ell *}, S_{\varepsilon}^{\ell *}\right)\right\}$ converges to the unique optimal solution (cf. Theorem 2.1), say, $\left(\bar{s}^{\ell}, \bar{S}^{\ell}\right)$, to $(\mathcal{C})_{(\bar{x}, \bar{X})}\left(\omega_{\ell}\right)$, for each $\ell \in \mathcal{L}$.

Consider next an arbitrary feasible solution $(x, X, \tilde{s}, \tilde{S})$ to the problem $\left(\mathcal{P}_{1}\right)$, and an arbitrary sequence $\left\{\left(x_{\varepsilon}, X_{\varepsilon}, \tilde{s}_{\varepsilon}, \tilde{S}_{\varepsilon}\right)\right\}$ of feasible solutions to the problems $\left(\mathcal{P}_{1}^{\varepsilon}\right)$, where however $\left\{\left(x_{\varepsilon}, X_{\varepsilon}\right)\right\} \rightarrow$ $(x, X)$. [For any given design $(x, X)$ satisfying the design constraints in $\left(\mathcal{P}_{1}\right)$, Proposition 1.1 .2 of Aubin and Frankowska $[\mathrm{AuF} 90]$ ensures the existence of a sequence $\left\{\left(x_{\varepsilon}, X_{\varepsilon}\right)\right\}$ of designs satisfying the design constraints in $\left(\mathcal{P}_{1}^{\varepsilon}\right)$.] Corollary 3.2 then implies that the sequence $\left\{\left(\tilde{s}_{\varepsilon}, \tilde{S}_{\varepsilon}\right)\right\}$ of states converges to the limit state $(\tilde{s}, \tilde{S})$.

We then have that

$$
\begin{aligned}
c^{f}(\bar{x}, \bar{X}, \bar{s}, \bar{S}) & \leq \liminf _{\varepsilon \rightarrow 0} c^{f}\left(x_{\varepsilon}^{*}, X_{\varepsilon}^{*}, \tilde{s}_{\varepsilon}^{*}, \tilde{S}_{\varepsilon}^{*}\right) \\
& \leq \liminf _{\varepsilon \rightarrow 0} c^{f}\left(x_{\varepsilon}, X_{\varepsilon}, \tilde{s}_{\varepsilon}, \tilde{S}_{\varepsilon}\right) \\
& \leq \lim _{\varepsilon \rightarrow 0} c^{f}\left(x_{\varepsilon}, X_{\varepsilon}, \tilde{s}, \tilde{S}\right) \\
& =c^{f}(x, X, \tilde{s}, \tilde{S}),
\end{aligned}
$$

where the inequalities follow from the lsc property of $c^{f}$, the optimality of $\left(x_{\varepsilon}^{*}, X_{\varepsilon}^{*}, \tilde{s}_{\varepsilon}^{*}, \tilde{S}_{\varepsilon}^{*}\right)$ and feasibility of $\left(x_{\varepsilon}, X_{\varepsilon}, \tilde{s}_{\varepsilon}, \tilde{S}_{\varepsilon}\right)$ in $\left(\mathcal{P}_{1}^{\varepsilon}\right)$, and the optimality of $\left(\tilde{s}_{\varepsilon}, \tilde{S}_{\varepsilon}\right)$ in $(\mathcal{C})_{\left(x_{\varepsilon}, X_{\varepsilon}\right)}$; finally, the equality follows from the continuity of $c^{f}(\cdot, \cdot, \bar{s}, \bar{S})$. By $(32),(\bar{x}, \bar{X}, \bar{s}, \bar{S})$ is optimal in $\left(\mathcal{P}_{1}\right)$. The convergence of the sequence $\left\{\left(x_{\varepsilon}^{*}, X_{\varepsilon}^{*}, \tilde{s}_{\varepsilon}^{*}, \tilde{S}_{\varepsilon}^{*}\right)\right\}$ to the optimal solution set of $\left(\mathcal{P}_{1}\right)$ then follows from its compactness. Since $c^{f}$ equals $c^{d}$ on $\operatorname{gr} \mathcal{S}_{\ell}, \ell \in \mathcal{L}$, the last result follows also.

As remarked above, it is computationally quite often preferable to work in the displacement space as compared to working in the force space when solving for an equilibrium. However, 
as the lower design bound tends to zero, it seems that the sequence $\left\{\left(u_{\varepsilon}^{*}, e_{\varepsilon}^{*}\right)\right\}$ of equilibrium displacements may be unbounded if the final design is such that the corresponding equilibrium displacement solution is unspecified along certain directions. This can be contrasted with the result of Theorem 3.2 which establishes that the sequence of equilibrium displacements tends to a minimum-energy equilibrium solution for the limit design provided that the design sequence tends strictly monotonically towards it. The reason for this perhaps surprising difference is that the optimal designs in the $\varepsilon$-perturbed problems need not tend strictly monotonically to a limit design; certain elements of the sequence $\left\{\left(x_{\varepsilon}^{*}, X_{\varepsilon}^{*}\right)\right\}$ may even converge finitely.

\subsection{The stress-constrained minimum weight problem}

\subsubsection{The design optimization model}

Let $\rho_{1}>0$ be the density of the structure material and $\rho_{2}>0$ the density of the cable material, and suppose that the effective stress is not allowed to exceed $\bar{\sigma}_{1}$ in the structure and $\bar{\sigma}_{2}$ in the cables. Since the effective stress in the structure is $\sigma_{i}^{e}=\left|\sigma_{i}\right|$, the bound in part $i$ can be expressed as

$$
x_{i}\left|\sigma_{i}\right| \leq \bar{\sigma}_{1} x_{i},
$$

where the factor $x_{i}$ has been introduced to "remove" the constraint when there is no material to carry any stress. Using (3) in (33), we get

$$
\left|s_{i}\right| \leq \bar{\sigma}_{1} x_{i}
$$

Consider now also the effect of introducing a stochastic load in this problem. The structural response depends on the stochastic variable $\omega$. In this formulation the state variable is represented by the internal forces $s$ and $S$. Previously we started with the deterministic problem and then replaced the state variable by its expected value. Proceeding similarly one gets:

$$
\left\{\begin{aligned}
\min _{(x, X, s, S)} & w(x, X):=\rho_{1} 1_{m}^{T} x+\rho_{2} 1_{r_{2}}^{T} X, \\
& \left\{\begin{array}{l}
\underline{x} \leq x \leq \bar{x}, \\
\underline{X} \leq X \leq \bar{X}, \\
E_{\omega}\left[\left|s_{i}(\omega)\right|\right] \leq \bar{\sigma}_{1} x_{i}, \quad i=1, \ldots, m, \\
E_{\omega}\left[S_{j}(\omega)\right] \leq \bar{\sigma}_{2} X_{j}, \quad j=1, \ldots, r_{2}, \\
(s, S)(\omega) \operatorname{solves}(\mathcal{C})_{(x, X)}(\omega), \quad \omega \in \Omega .
\end{array}\right.
\end{aligned}\right.
$$

(We allow for the vectors $\bar{x}$ and $\bar{X}$ to take on infinite values.) It makes more sense to use $E_{\omega}\left[\left|s_{i}(\omega)\right|\right]$ instead of $\left|E_{\omega}\left[s_{i}(\omega)\right]\right|$ since it is more conservative. (One can get $\left|E_{\omega}\left[s_{i}(\omega)\right]\right|=0$ even if the stresses are very large for all events.)

Discretizing $\Omega$ into $\Omega=\left\{\omega_{1}, \ldots, \omega_{|\mathcal{L}|}\right\}$ and using Simpson's rule as before,

$$
E_{\omega}\left[\left|s_{j}(\omega)\right|\right]=\int_{\underline{\omega}}^{\bar{\omega}}\left|s_{j}(\omega)\right| p(\omega) d \omega \approx \sum_{i=1}^{N-1}\left|s_{j}\left(\omega_{i}\right)\right| p\left(\omega_{i}\right) \frac{h_{i}+h_{i+1}}{2}=\sum_{\ell \in \mathcal{L}} \rho_{\ell}\left|s_{j}^{\ell}\right|,
$$

where $s_{j}^{\ell}=s_{j}\left(\omega_{\ell}\right)$, and, again, $\rho_{\ell}=p\left(\omega_{\ell}\right)\left(h_{\ell}+h_{\ell+1}\right) / 2$. In the same manner,

$$
E_{\omega}\left[S_{j}(\omega)\right] \approx \sum_{\ell \in \mathcal{L}} \rho_{\ell} S_{j}^{\ell}
$$


and therefore we arrive at the optimization problem

$$
\begin{cases}\min _{(x, X, \tilde{s}, \tilde{S})} & w(x, X):=\rho_{1} 1_{m}^{T} x+\rho_{2} 1_{r_{2}}^{T} X, \\
& \left\{\begin{array} { l } 
{ \underline { x } \leq x \leq \overline { x } , } \\
{ \underline { X } \leq X \leq \overline { X } } \\
{ \text { s.t. } }
\end{array} \quad \left\{\begin{array}{l}
\sum_{\ell \in \mathcal{L}} \rho_{\ell}\left|s_{i}^{\ell}\right| \leq \bar{\sigma}_{1} x_{i}, \quad i=1, \ldots, m, \\
\sum_{\ell \in \mathcal{L}} \rho_{\ell} S_{j}^{\ell} \leq \bar{\sigma}_{2} X_{j}, \quad j=1, \ldots, r_{2}, \\
\left(s^{\ell}, S^{\ell}\right) \operatorname{solves}(\mathcal{C})_{(x, X)}\left(\omega_{\ell}\right), \quad \ell \in \mathcal{L} .
\end{array}\right.\right.\end{cases}
$$

Instead of taking the expected value, we can ensure that the stresses are below the bounds for all $\omega \in \Omega$ by adding side constraints. This was not an option in the same way before, where the state variable appeared in the objective function. ${ }^{3}$ One arrives finally at

$$
\left(\mathcal{P}_{2}\right) \begin{cases}\min _{(x, X, \tilde{s}, \tilde{S})} & w(x, X):=\rho_{1} 1_{m}^{T} x+\rho_{2} 1_{r_{2}}^{T} X, \\
& \left\{\begin{array}{l}
\underline{x} \leq x \leq \bar{x}, \\
\underline{X} \leq X \leq \bar{X}, \\
\left|s_{i}^{\ell}\right| \leq \bar{\sigma}_{1} x_{i}, \quad i=1, \ldots, m, \quad \ell \in \mathcal{L}, \\
S_{j}^{\ell} \leq \bar{\sigma}_{2} X_{j}, \quad j=1, \ldots, r_{2}, \quad \ell \in \mathcal{L}, \\
\left(s^{\ell}, S^{\ell}\right) \operatorname{solves}(\mathcal{C})_{(x, X)}\left(\omega_{\ell}\right), \quad \ell \in \mathcal{L} .
\end{array}\right.\end{cases}
$$

This is a more conservative model, and the number of side constraints is in general very large. This formulation looks like the traditional worst-case multiple load-case formulation for the stressconstrained minimum weight problem.

\subsubsection{Existence of optimal designs}

Theorem 4.3 (Existence of optimal solutions to $\left(\mathcal{P}_{2}\right)$ ). Suppose the feasible set $\mathcal{F}^{\mathcal{P}_{2}}$ of $\left(\mathcal{P}_{2}\right)$ is nonempty. Then, there exists at least one optimal solution to $\left(\mathcal{P}_{2}\right)$.

Proof. We begin by constructing a global upper bound on the energy function $\mathcal{E}^{\ell}\left(x, X, s^{\ell}, S^{\ell}\right)$, $\ell \in \mathcal{L}$, defined in the objective of $\left(\mathcal{P}_{1}\right)$, over $\mathcal{F}^{\mathcal{P}_{2}}$. Consider a feasible design $(x, X)$. Since the constraints on $\left(s^{\ell}, S^{\ell}\right)$ are linear and $\mathcal{E}^{\ell}(x, X, \cdot, \cdot)$ is convex, the maximum, if it exists, is attained at an extreme point (e.g., [BSS93, Theorem 3.4.7]). An upper bound of this value is obtained by considering only the stress constraints, as follows.

Each term in $\frac{\left(s_{i}^{\ell}\right)^{2}}{E x_{i}}, i \in \mathcal{I}(x)$, when maximized with respect to $s_{i}^{\ell}$ over the set $\left|s_{i}^{\ell}\right| \leq \bar{\sigma}_{1} x_{i}$, attains its maximum at $s_{i}^{\ell}= \pm \bar{\sigma}_{1} x_{i}$.

Each term in $\frac{\left(L_{j} S_{j}^{\ell}\right)^{2}}{2 E_{c} X_{j}}+\left(g_{2}\right)_{j} S_{j}^{\ell}, j \in \mathcal{J}(X)$, is either maximal at $S_{j}^{\ell}=0$ or at $S_{j}^{\ell}=\bar{\sigma}_{2} X_{j}$.

Hence, we obtain that for any feasible $(x, X)$ and $\ell \in \mathcal{L}$,

$$
\mathcal{E}^{\ell}\left(x, X, s^{\ell}, S^{\ell}\right) \leq \frac{1}{2} \sum_{i \in \mathcal{I}(x)} x_{i}\left(\bar{\sigma}_{1}\right)^{2} E^{-1}+\sum_{j \in \mathcal{J}(X)} \max \left\{0,\left(\frac{X_{j}\left(L_{j} \bar{\sigma}_{2}\right)^{2}}{2 E_{c}}+\left(g_{2}\right)_{j} \bar{\sigma}_{2} X_{j}\right)\right\} .
$$

\footnotetext{
${ }^{3}$ This is true of course if we refrain from modelling our problem as a multi-objective optimization problem. The side constraints can alternatively be written as $\max _{\ell \in \mathcal{L}}\left\{\left|s_{i}^{\ell}\right|\right\} \leq \bar{\sigma}_{1} x_{i}, i=1, \ldots, m$, whence the analogous modification in the compliance problem leads to a min-max formulation.
} 
Since the objective is to minimize the (strictly positive) weighted sum of the total weights, and the design is nonnegative, there exists an upper bound $(\widehat{x}, \widehat{X}) \leq(\bar{x}, \bar{X})$ on the design vector $(x, X)$ such that no candidate for an optimum exceeds $(\widehat{x}, \widehat{X})$. If we add the constraints

$$
\begin{aligned}
\mathcal{E}^{\ell}\left(x, X, s^{\ell}, S^{\ell}\right) & \leq \frac{1}{2} \sum_{i=1}^{m} \widehat{x}_{i}\left(\bar{\sigma}_{1}\right)^{2} E^{-1}+\sum_{j=1}^{r_{2}} \max _{j} \leq X_{j} \leq \widehat{X}_{j} \\
& =: \nu_{\ell}<\infty, \quad \ell \in \mathcal{L},
\end{aligned}
$$

we then add constraints which are redundant in the problem $\left(\mathcal{P}_{2}\right)$. Moreover, according to Corollary 3.1, the sets $\operatorname{gr} \mathcal{S}_{\ell} \cap L_{\nu_{\ell}}^{\mathcal{E}^{\ell}}$ are closed. Hence, since the rest of the constraints are defined by continuous functions in $\left(x, X, s^{\ell}, S^{\ell}\right)$, the feasible set of $\left(\mathcal{P}_{2}\right)$ is closed, as well as nonempty. The upper-level objective function is continuous, and coercive since the set of candidate designs $(x, X)$ is bounded and the same is true for the set of equilibrium forces $\left(s^{\ell}, S^{\ell}\right), \ell \in \mathcal{L}$, thanks to the stress constraints. Hence, Weierstrass' Theorem applies.

\subsection{3 $\varepsilon$-perturbation}

In the case of a topology optimization, where $(\underline{x}, \underline{X})=(0,0)$, for computational reasons we need to add small positive design bounds. Cheng and Guo [ChG97] have however proven that the naive approach - replacing the zero design bounds by $\varepsilon 1_{m} \leq x$ and $\varepsilon 1_{r_{2}} \leq X$ for some small positive value of $\varepsilon$-will in general not generate solutions that are close to the desired ones in stress-constrained problems. They therefore suggested another $\varepsilon$-relaxation where also the stress constraints are modified. Generalizing this procedure somewhat, and introducing the function $o: \mathbb{R}_{++} \mapsto \mathbb{R}_{++}$to be continuous and such that $\{o(\varepsilon) / \varepsilon\}$ tends to zero while $\left\{o(\varepsilon) / \varepsilon^{2}\right\}$ is bounded away from zero, as $\varepsilon$ tends to zero, we arrive at

$$
\left(\mathcal{P}_{2}^{\varepsilon}\right) \begin{cases}\min _{(x, X, \tilde{s}, \tilde{S})} & w(x, X):=\rho_{1} 1_{m}^{T} x+\rho_{2} 1_{r_{2}}^{T} X, \\
\text { s.t. } & \left\{\begin{array}{l}
o(\varepsilon) 1_{m} \leq x \leq \bar{x}+o(\varepsilon) 1_{m}, \\
o(\varepsilon) 1_{r_{2}} \leq X \leq \bar{X}+o(\varepsilon) 1_{r_{2}}, \\
\left|s_{i}^{\ell}\right| \leq \bar{\sigma}_{1} x_{i}+\varepsilon, \quad i=1, \ldots, m, \quad \ell \in \mathcal{L}, \\
S_{i}^{\ell} \leq \bar{\sigma}_{2} X_{i}+\varepsilon, \quad i=1, \ldots, r_{2}, \quad \ell \in \mathcal{L}, \\
\left(s^{\ell}, S^{\ell}\right) \operatorname{solves}(\mathcal{C})_{(x, X)}\left(\omega_{\ell}\right), \quad \ell \in \mathcal{L} .
\end{array}\right.\end{cases}
$$

Similarly to Theorem 4.2, we establish that this problem indeed gives solutions close to the ones of $\left(\mathcal{P}_{2}\right)$.

Theorem 4.4 (Convergence of $\varepsilon$-perturbed solutions). Suppose the feasible set $\mathcal{F}^{\mathcal{P}_{2}}$ of $\left(\mathcal{P}_{2}\right)$ is nonempty. For each $\varepsilon>0$, let $\left(x_{\varepsilon}^{*}, X_{\varepsilon}^{*}, \tilde{s}_{\varepsilon}^{*}, \tilde{S}_{\varepsilon}^{*}\right)$ denote an arbitrary optimal solution to $\left(\mathcal{P}_{2}^{\varepsilon}\right)$. Then, the sequence $\left\{\left(x_{\varepsilon}^{*}, X_{\varepsilon}^{*}, \tilde{s}_{\varepsilon}^{*}, \tilde{S}_{\varepsilon}^{*}\right)\right\}$ is bounded, and converges to the optimal solution set $\mathrm{SOL}\left(\mathcal{P}_{2}\right)$ of $\left(\mathcal{P}_{2}\right)$, in the sense that

$$
\left\{\min _{(x, X, \tilde{s}, \tilde{S}) \in \operatorname{SOL}\left(\mathcal{P}_{2}\right)}\left\|\left(x_{\varepsilon}^{*}, X_{\varepsilon}^{*}, \tilde{s}_{\varepsilon}^{*}, \tilde{S}_{\varepsilon}^{*}\right)-(x, X, \tilde{s}, \tilde{S})\right\|\right\} \rightarrow 0 .
$$

Moreover, $\left\{w\left(x_{\varepsilon}^{*}, X_{\varepsilon}^{*}\right)\right\}$ converges to the optimal value of $\left(\mathcal{P}_{2}\right)$.

Proof. Similarly to the proof of Theorem 4.3, we will establish that the energy functionals $\mathcal{E}^{\ell}$ are bounded on the feasible set of the problem $\left(\mathcal{P}_{2}^{\varepsilon}\right)$ for any $\varepsilon>0$, by introducing an upper 
design bound $\left(\widehat{x}_{\varepsilon}, \widehat{X}_{\varepsilon}\right)$ and the upper bound $\nu_{\ell, \varepsilon}<\infty$. [In fact, it is sufficient to take $\left(\widehat{x}_{\varepsilon}, \widehat{X}_{\varepsilon}\right)=$ $(\widehat{x}+o(\varepsilon), \widehat{X}+o(\varepsilon))$.] Using the relaxed stress constraints, the inequality $(a+b)^{2} \leq 2 a^{2}+2 b^{2}$ (for any $a, b \in \mathbb{R})$, and $x_{i}, X_{i} \geq o(\varepsilon)$, one has

$$
\begin{aligned}
\mathcal{E}^{\ell}\left(x, X, s^{\ell}, S^{\ell}\right) & \leq \sum_{i \in \mathcal{I}(x)} \frac{\left(\bar{\sigma}_{1} x_{i}+\varepsilon\right)^{2}}{2 E x_{i}}+\sum_{j \in \mathcal{J}(X)}\left(\frac{L_{j}^{2}\left(\bar{\sigma}_{2} X_{j}+\varepsilon\right)^{2}}{2 E_{c} X_{j}}+\left|\left(g_{2}\right)_{j}\right|\left(\bar{\sigma}_{2} X_{j}+\varepsilon\right)\right) \\
& \leq \sum_{i \in \mathcal{I}(x)}\left(\frac{\bar{\sigma}_{1}^{2} x_{i}}{E}+\frac{\varepsilon^{2}}{E x_{i}}\right)+\sum_{j \in \mathcal{J}(X)}\left(\frac{L_{j}^{2} \bar{\sigma}_{2}^{2} X_{j}}{E_{c}}+\frac{\varepsilon^{2} L_{j}^{2}}{E_{c} X_{j}}+\left|\left(g_{2}\right)_{j}\right|\left(\bar{\sigma}_{2} X_{j}+\varepsilon\right)\right) \\
& \leq \sum_{i=1}^{m}\left(\frac{\bar{\sigma}_{1}^{2}\left(\widehat{x}_{\varepsilon}\right)_{i}}{E}+\frac{1}{E o(\varepsilon) / \varepsilon^{2}}\right) \\
& +\sum_{j=1}^{r_{2}}\left(\frac{L_{j}^{2} \bar{\sigma}_{2}^{2}\left(\widehat{X}_{\varepsilon}\right)_{j}}{E_{c}}+\frac{L_{j}^{2}}{E_{c} O(\varepsilon) / \varepsilon^{2}}+\left|\left(g_{2}\right)_{j}\right|\left(\bar{\sigma}_{2}\left(\widehat{X}_{\varepsilon}\right)_{j}+\varepsilon\right)\right) \\
& =: \nu_{\ell, \varepsilon}<\infty, \quad \ell \in \mathcal{L},
\end{aligned}
$$

where $\nu_{\ell, \varepsilon}$ are bounded as $\varepsilon \rightarrow 0$ since $\left\{o(\varepsilon) / \varepsilon^{2}\right\}$ stays bounded away from zero. We conclude that the sequence $\left\{\left(x_{\varepsilon}^{*}, X_{\varepsilon}^{*}, \tilde{s}_{\varepsilon}^{*}, \tilde{S}_{\varepsilon}^{*}\right)\right\}$ is bounded. Suppose that $\left(x^{*}, X^{*}, \tilde{s}^{*}, \tilde{S}^{*}\right)$ is a limit point of this sequence. Invoking Theorems 2.2 and 3.1 then yields that $\left(\tilde{s}^{*}, \tilde{S}^{*}\right)$ is the optimal solution to $(\mathcal{C})_{\left(x^{*}, X^{*}\right)}\left(\omega_{\ell}\right), \ell \in \mathcal{L}$. The continuity properties of the other constraints of the problem $\left(\mathcal{P}_{2}\right)$ then imply that $\left(x^{*}, X^{*}, \tilde{s}^{*}, \tilde{S}^{*}\right)$ is feasible in $\left(\mathcal{P}_{2}\right)$. Let $(x, X, \tilde{s}, \tilde{S})$ be an arbitrary feasible solution to $\left(\mathcal{P}_{2}\right)$, set $x_{\varepsilon}=x+o(\varepsilon) 1_{m}, X_{\varepsilon}=X+o(\varepsilon) 1_{r_{2}}$ and let $\left(s_{\varepsilon}^{\ell}, S_{\varepsilon}^{\ell}\right)$ solve $(\mathcal{C})_{\left(x_{\varepsilon}, X_{\varepsilon}\right)}\left(\omega_{\ell}\right), \ell \in \mathcal{L}$.

We then have, by Theorem 3.3, that for some $\kappa>0$ and every $i=1, \ldots, m$ and $\ell \in \mathcal{L}$,

$$
\left|\left(s_{\varepsilon}^{\ell}\right)_{i}\right| \leq \kappa o(\varepsilon)+\left|s_{i}^{\ell}\right| \leq \kappa o(\varepsilon)+\bar{\sigma}_{1}\left[x_{i}-o(\varepsilon)+o(\varepsilon)\right]=\left(\kappa-\bar{\sigma}_{1}\right) o(\varepsilon)+\bar{\sigma}_{1}\left(x_{\varepsilon}\right)_{i} \leq \bar{\sigma}_{1}\left(x_{\varepsilon}\right)_{i}+\varepsilon,
$$

for all $\varepsilon$ small enough, where we have used the assumption that $\{o(\varepsilon) / \varepsilon\} \rightarrow 0$. $S_{\varepsilon}$ can be treated in the same way, and since clearly $\left(x_{\varepsilon}, X_{\varepsilon}\right)$ satisfies the design constraints, $\left(x_{\varepsilon}, X_{\varepsilon}, \tilde{s}_{\varepsilon}, \tilde{S}_{\varepsilon}\right)$ is permissible in $\left(\mathcal{P}_{2}^{\varepsilon}\right)$. Hence, $w\left(x_{\varepsilon}^{*}, X_{\varepsilon}^{*}\right) \leq w\left(x_{\varepsilon}, X_{\varepsilon}\right)$. Letting $\varepsilon$ tend to zero in this inequality, we obtain that $w\left(x^{*}, X^{*}\right) \leq w(x, X)$, whence we may conclude that $\left(x^{*}, X^{*}, \tilde{s}^{*}, \tilde{S}^{*}\right)$ solves $\left(\mathcal{P}_{2}\right)$. The result then follows from the compactness of $\operatorname{SOL}\left(\mathcal{P}_{2}\right)$ and the continuity of $w$.

\subsubsection{Example: One-bar truss with a cable revisited}

Consider again the one-dimensional structure in Figure 1. Assume that allowable stresses are $\bar{\sigma}_{1}=1$ and $\bar{\sigma}_{2}=1 / 2$ for the bar and cable material, respectively. Invoking the stress constraints $|s| \leq x$ and $S \leq X / 2$, the nested version of $\left(\mathcal{P}_{2}\right)$ with $\underline{x}=\underline{X}=0$ becomes

$$
\begin{cases}\min _{(x, X)} & x+X \\
\text { s.t. } & \left\{\begin{array}{l}
x \geq 0, X \geq 0, \\
x \leq(x+X) x \\
X \leq(x+X) X / 2
\end{array}\right.\end{cases}
$$

recalling (19). We presume the upper design bounds are passive. The admissible domain is shown in Figure 2.

One sees immediately from the figure that the optimal solution is

$$
x^{*}=1, \quad X^{*}=0, \quad \text { with optimal weight }=x^{*}+X^{*}=1 .
$$


When this type of optimal solution and design domain appears, in the structural optimization community one talks about the "stress singularity phenomenon", and the optimal solution is sometimes referred to as a "singular topology". The first example of a singular topology was reported by Sved and Ginos [SvG68], and design domains very similar to the one in Figure 2 were presented by Kirsch [Kir90] and later also by Rozvany and Birker [RoB94] and by Cheng [Che95].

From a mathematical programming point of view, the singularity stems from the constraints not being qualified (in the sense of, for example, Slater), cf. [BSS93, DuB98]. This implies that the Karush-Kuhn-Tucker (KKT) conditions need not be necessary for (local) optimality. [Obviously, the nonconvexity of the problem adds to this the fact that the KKT conditions neither are sufficient for (local) optimality.] Almost every practical numerical algorithm generates solutions that are only guaranteed to fulfill the KKT conditions [e.g., $(x, X)=(2,0)$ in Figure 2], which, hence, need not have anything to do with the desired global (or even local) minima. If small strictly positive lower design bounds are enforced, that is, $\underline{x}=\underline{X}=\varepsilon$, then one gets the incorrect "optimal" weight 2 , and the optimal solutions $\left(x_{\varepsilon}^{*}, X_{\varepsilon}^{*}\right)$ satisfy

$$
\left\|\left(x^{*}, X^{*}\right)-\left(x_{\varepsilon}^{*}, X_{\varepsilon}^{*}\right)\right\| \geq \frac{1}{\sqrt{2}}, \quad \forall \varepsilon \in(0,1),
$$

so the straightforward $\varepsilon$-perturbation is clearly incorrect.

In this example, the stress bounds were chosen differently in the bar and cable. When the elastic modulii, specific weights and stress bounds are uniform, then, for a single load, the problem $\left(\mathcal{P}_{2}\right)$ seems to provide no major complications; in fact optimal solutions can, at least in the non-unilateral case, be obtained by solving a dual pair of linear programs [DGG64, Sva94]. However, since it is very plausible that one wishes to use cable and bar materials that are not the same, or that they are subject to different allowable stresses, one must deal with this type of problem. Moreover, Stolpe and Svanberg [StS01] showed that multiple loads (while keeping the other data uniform) suffice to produce difficult problem instances, where global optima are situated at degenerate parts of the feasible domain. Since multiple load-cases appear after discretization of the probability space, this means that one can expect the optimal solution to be singular, even if the elastic modulii, specific weights and stress bounds are uniform.

Let us now consider the example in view of the $\varepsilon$-relaxed (or, $\varepsilon$-perturbed) statement $\left(\mathcal{P}_{2}^{\varepsilon}\right)$. We take $o(\varepsilon):=\varepsilon^{2}$, so $\underline{x}=\underline{X}=\varepsilon^{2}$, presume the upper bounds to be passive, and write the stress constraints on the form

$$
|s| \leq x+\varepsilon, \quad S \leq X / 2+\varepsilon .
$$

Then the nested version of $\left(\mathcal{P}_{2}^{\varepsilon}\right)$ becomes

$$
\begin{cases}\min _{(x, X)} & x+X, \\
\text { s.t. } & \left\{\begin{array}{l}
x \geq \varepsilon^{2}, X \geq \varepsilon^{2}, \\
x \leq(x+X)(x+\varepsilon), \\
X \leq(x+X)(X / 2+\varepsilon) .
\end{array}\right.\end{cases}
$$

The admissible domain for $\varepsilon=0.1$ is shown in Figure 5 .

The optimal solution, for a general $\varepsilon>0$, is given by

$$
x_{\varepsilon}^{*}=\frac{1-5 \varepsilon+\sqrt{1-2 \varepsilon+9 \varepsilon^{2}}}{2}, \quad X_{\varepsilon}^{*}=\frac{x_{\varepsilon}^{*}}{x_{\varepsilon}^{*}+\varepsilon}-x_{\varepsilon}^{*},
$$

and the optimal weight is $x_{\varepsilon}^{*} /\left(x_{\varepsilon}^{*}+\varepsilon\right)$. Clearly,

$$
\lim _{\varepsilon \rightarrow 0} x_{\varepsilon}^{*}=x^{*}, \quad \lim _{\varepsilon \rightarrow 0} X_{\varepsilon}^{*}=X^{*},
$$

as Theorem 4.4 predicts. 


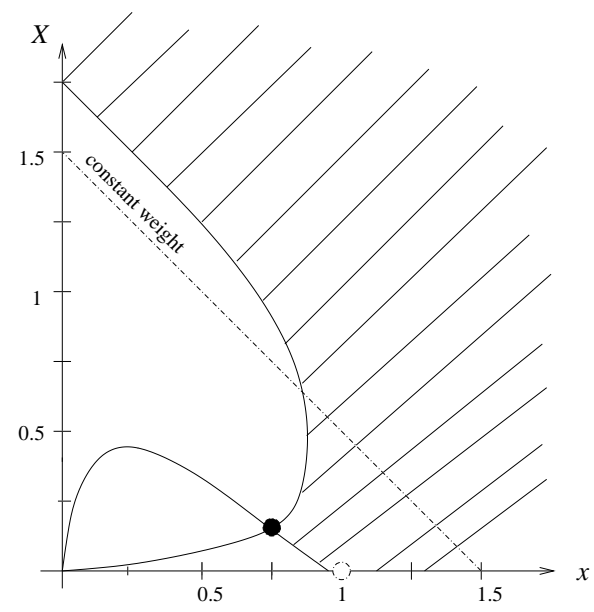

Figure 5: The admissible design domain for $\varepsilon=0.1$. The optimal solution is at the black circle.

\section{FE-discretized sheets in contact}

\subsection{The design optimization model}

Consider a family of linearly elastic continua occupying a region $\mathcal{B}(h)$ in space,

$$
\mathcal{B}(h)=\left\{\left(\xi_{1}, \xi_{2}, \xi_{3}\right) \in \mathbb{R}^{3}\left|\quad\left(\xi_{1}, \xi_{2}\right) \in \hat{\Omega},\right| \xi_{3} \mid<h\left(\xi_{1}, \xi_{2}\right) / 2\right\}
$$

where $\hat{\Omega}$ is a fixed open and plane two-dimensional set and $h: \hat{\Omega} \rightarrow \mathbb{R}_{+}$is a thickness function. Note that the topology of $\mathcal{B}(h)$ can be changed by introducing holes through setting $h\left(\xi_{1}, \xi_{2}\right)=0$.

We assume that $\mathcal{B}(h)$ is loaded in such a way that the state of stress is plane. The design objective now is to minimize the (in some sense) effective stress $\sigma_{M}: \mathcal{B}(h) \rightarrow \mathbb{R}_{+}$. It would have been desirable to minimize the maximum effective stress, that is, $\left\|\sigma_{M}\right\|_{L^{\infty}(\mathcal{B}(h))}$, but since $\sigma_{M}$ is only guaranteed to belong to $L^{2}$, we instead choose

$$
\begin{array}{rlr}
\left\|\sigma_{M}\right\|_{L^{2}(\mathcal{B}(h))}^{2} & =\int_{\mathcal{B}(h)} \sigma_{M}^{2} d \mathcal{B} \\
& =\int_{\hat{\Omega}} \int_{-h\left(\xi_{1}, \xi_{2}\right) / 2}^{h\left(\xi_{1}, \xi_{2}\right) / 2} \sigma_{M}^{2} d \xi_{3} d \xi_{1} d \xi_{2} \\
& =\int_{\hat{\Omega}} h \sigma_{M}^{2} d \xi_{1} d \xi_{2} & \text { (plane stress) } \\
& \approx \sum_{i=1}^{m} h_{i} A_{i}\left(\sigma_{i}^{e}\right)^{2} & \quad \text { (FE-discretization step) } \\
& =\sum_{i \in \mathcal{I}(x)} x_{i} \sigma_{i}^{T} M \sigma_{i}, & \left(x_{i}\right. \text { is the material volume) }
\end{array}
$$

where $A_{i}$ is the area of the finite element, $\sigma_{i}^{T}=\left(\sigma_{x}, \sigma_{y}, \tau_{x y}\right)$, and $M$ is a positive definite and symmetric matrix, cf. Section 2.1. Finally, using (3), we get

$$
\left\|\sigma_{M}\right\|_{L^{2}(\mathcal{B}(h))}^{2} \approx \sum_{i \in \mathcal{I}(x)} \frac{s_{i}^{T} M s_{i}}{x_{i}} .
$$


We assume that the unilateral constraints are due to rigid supports, so no cables are present, that is, the variables $(S, e)$ do not appear in the equilibrium problems and $x$ is the only design variable. The energy function hence now takes the form

$$
\mathcal{E}(x, s, \lambda)=\frac{1}{2} \sum_{i \in \mathcal{I}(x)} \frac{s_{i}^{T} E^{-1} s_{i}}{x_{i}}+g_{1}^{T} \lambda
$$

whenever it is finite. If the initial gaps are zero, that is, if $g_{1}=0$, then compliance is equal to $\frac{1}{2} \sum_{i \in \mathcal{I}(x)} \frac{s_{i}^{T} E^{-1} s_{i}}{x_{i}}$, which is obtained in (35) by choosing $M=\frac{1}{2} E^{-1}$. By choosing the matrix given in Section 2.1 one minimizes the $L^{2}$-norm of the von Mises effective stress. We enforce an upper bound on the available material volume, $\int_{\mathcal{B}(h)} d \mathcal{B} \leq v$. Further,

$$
\begin{aligned}
\int_{\mathcal{B}(h)} d \mathcal{B} & =\int_{\hat{\Omega}} \int_{-h\left(\xi_{1}, \xi_{2}\right) / 2}^{h\left(\xi_{1}, \xi_{2}\right) / 2} d \xi_{3} d \xi_{1} d \xi_{2} \\
& =\int_{\hat{\Omega}} h d \xi_{1} d \xi_{2} \\
& \approx \sum_{i=1}^{m} h_{i} A_{i} \quad \text { (FE-discretization step) } \\
& =\sum_{i=1}^{m} x_{i},
\end{aligned}
$$

so the constraint becomes $1_{m}^{T} x \leq v$. Including stochastic loads and performing a discretization of the probability space as before, we can pose the following problem statement:

$$
\left(\mathcal{P}_{3}\right) \begin{cases}\min _{(x, \tilde{s}, \tilde{\lambda})} & c^{f, M}(x, \tilde{s}):=\sum_{\ell \in \mathcal{L}} \rho_{\ell} \sum_{i \in \mathcal{I}(x)} \frac{\left(s_{i}^{\ell}\right)^{T} M s_{i}^{\ell}}{x_{i}}, \\
\text { s.t. } & \left\{\begin{array}{l}
1_{m}^{T} x \leq v, \quad x \geq 0, \\
\left(s^{\ell}, \lambda^{\ell}\right) \operatorname{solves}(\mathcal{C})_{(x)}\left(\omega_{\ell}\right), \quad \ell \in \mathcal{L} .
\end{array}\right.\end{cases}
$$

For more details on how to arrive at the mathematical programming problem by FE-discretization, we refer to [PeH98].

\subsection{Existence of optimal designs}

The following result is proven similarly to Theorem 4.1.

Theorem 5.1 (Existence of optimal solutions to $\left(\mathcal{P}_{3}\right)$ ). Suppose the feasible set $\mathcal{F}^{\mathcal{P}_{3}}$ of $\left(\mathcal{P}_{3}\right)$ is nonempty. Then, there exists at least one optimal solution to $\left(\mathcal{P}_{3}\right)$.

Proof. Since $c^{f, M}$ is to be minimized, we can presume that $\mathcal{F}^{\mathcal{P}_{3}}$ is such that $c^{f, M}$ is proper with respect to $\mathcal{F}^{\mathcal{P}_{3}}$, and even bounded from above on $\mathcal{F}^{\mathcal{P}_{3}}$ (or construct it as such, by introducing an additional redundant constraint of the form $c^{f, M}(x, \tilde{s}) \leq \nu$, with $\left.\nu<\infty\right)$. Matrix norms being essentially equivalent, we obtain, for some $\kappa>0$, that

$$
\begin{aligned}
\sum_{\ell \in \mathcal{L}} \rho_{\ell} \mathcal{E}^{\ell}\left(x, s^{\ell}, \lambda^{\ell}\right) & \leq \kappa c^{f, M}(x, \tilde{s})+\sum_{\ell \in \mathcal{L}} \rho_{\ell} g_{1}^{T} \lambda^{\ell} \\
& =\kappa c^{f, M}(x, \tilde{s})+\sum_{\ell \in \mathcal{L}} \rho_{\ell} g_{1}^{T} C_{1}\left[f^{\ell}-B^{T} s^{\ell}\right]
\end{aligned}
$$


holds for all $(x, \tilde{s}, \tilde{\lambda}) \in \mathcal{F}^{\mathcal{P}_{3}}$, where the equality stems from the quasi-orthogonality of $C_{1}$ and the equilibrium conditions. This implies that without any loss of generality we may add constraints of the form

$$
\mathcal{E}^{\ell}\left(x, s^{\ell}, \lambda^{\ell}\right) \leq \nu_{\ell}+\left(a^{\ell}\right)^{T} s^{\ell}, \quad \ell \in \mathcal{L},
$$

to the constraints of the problem $\left(\mathcal{P}_{3}\right)$, for some appropriate choice of $\left(a^{\ell}, \nu_{\ell}\right)$. By the lsc property of the energy functional, the corresponding set is closed. Due to the relation (37), and Corollary 3.1, the intersection of these new constraints with the graphs $\operatorname{gr} \mathcal{S}_{\ell}, \ell \in \mathcal{L}$, is nonempty and closed. Hence, the feasible set of $\left(\mathcal{P}_{3}\right)$ is nonempty and closed. The upper-level objective function is (again by assumption) proper, and it is further lsc and coercive, since it is coercive in $s(M$ being positive definite, cf. further Lemma 3.2 and the proof of Theorem 2.1) and the feasible set in terms of $x$ is bounded. Hence, Weierstrass' Theorem applies.

\section{$5.3 \varepsilon$-perturbation}

When perturbing the problem by enforcing a volume lower bound $\varepsilon>0,(\mathcal{P})_{(x)}$ is always uniquely solvable, so we can switch from $(\mathcal{C})_{(x)}$ to $(\mathcal{P})_{(x)}$. Then we have to note that, by using $(4)$, the design objective becomes

$$
\left\|\sigma_{M}\right\|_{L^{2}(\mathcal{B}(h))}^{2} \approx \sum_{i=1}^{m} x_{i} u^{T} B_{i}^{T} E M E B_{i} u
$$

Therefore, by setting $\bar{M}_{i}=B_{i}^{T} E M E B_{i}$, we can pose

$$
\left(\mathcal{P}_{3}^{\varepsilon}\right) \begin{cases}\min _{(x, \tilde{u})} & c^{d, M}(x, \tilde{u}):=\sum_{\ell \in \mathcal{L}} \rho_{\ell} \sum_{i=1}^{m} x_{i}\left(u^{\ell}\right)^{T} \bar{M}_{i} u^{\ell}, \\
\text { s.t. } \quad\left\{\begin{array}{l}
1_{m}^{T} x \leq v+\varepsilon m, \quad x \geq \varepsilon 1_{m}, \\
u^{\ell} \text { solves }(\mathcal{P})_{(x)}\left(\omega_{\ell}\right), \quad \ell \in \mathcal{L} .
\end{array}\right.\end{cases}
$$

Similarly to Theorem 4.2, we establish that this problem indeed gives optimal solutions close to the ones of $\left(\mathcal{P}_{3}\right)$.

Theorem 5.2 (Convergence of $\varepsilon$-perturbed solutions). Suppose the feasible set $\mathcal{F}^{\mathcal{P}_{3}}$ of $\left(\mathcal{P}_{3}\right)$ is nonempty. For each $\varepsilon>0$, let $\left(x_{\varepsilon}^{*}, \tilde{u}_{\varepsilon}^{*}, \tilde{s}_{\varepsilon}^{*}, \tilde{\lambda}_{\varepsilon}^{*}\right)$ denote an arbitrary optimal solution to $\left(\mathcal{P}_{3}^{\varepsilon}\right)$. Then, the sequence $\left\{\left(x_{\varepsilon}^{*}, \tilde{s}_{\varepsilon}^{*}, \tilde{\lambda}_{\varepsilon}^{*}\right)\right\}$ is bounded, and converges to the optimal solution set $\mathrm{SOL}\left(\mathcal{P}_{3}\right)$ of $\left(\mathcal{P}_{3}\right)$, in the sense that

$$
\left\{\min _{(x, \tilde{s}, \tilde{\lambda}) \in \operatorname{SOL}\left(\mathcal{P}_{3}\right)}\left\|\left(x_{\varepsilon}^{*}, \tilde{s}_{\varepsilon}^{*}, \tilde{\lambda}_{\varepsilon}^{*}\right)-(x, \tilde{s}, \tilde{\lambda})\right\|\right\} \rightarrow 0 .
$$

Moreover, $\left\{c^{f, M}\left(x_{\varepsilon}^{*}, \tilde{s}_{\varepsilon}^{*}\right)\right\}$ and $\left\{c^{d, M}\left(x_{\varepsilon}^{*}, \tilde{u}_{\varepsilon}^{*}\right)\right\}$ converge to the optimal value of $\left(\mathcal{P}_{3}\right)$.

Proof. According to Theorem 5.1, an optimal solution exists to the problem $\left(\mathcal{P}_{3}^{\varepsilon}\right)$ for every $\varepsilon>0$ (since also $c^{d, M}$ is coercive whenever $\varepsilon>0$ ), as well as to the problem $\left(\mathcal{P}_{3}\right)$. Consider first the sequence $\left\{x_{\varepsilon}^{*}\right\}$. Clearly, this sequence is bounded since the feasible sets of $\left(\mathcal{P}_{3}^{\varepsilon}\right)$ in $x_{\varepsilon}$ are bounded, as well as that in $x$ of $\left(\mathcal{P}_{3}\right)$. The sequence $\left\{c^{f, M}\left(x_{\varepsilon}^{*}, \tilde{s}_{\varepsilon}^{*}\right)\right\}$ is bounded as well. To see this, consider, for any $\varepsilon>0$, an optimal solution, say $\left(\hat{x}_{\varepsilon}^{*}, \hat{s}_{\varepsilon}^{*}\right)$, to the problem which equals $\left(\mathcal{P}_{3}^{\varepsilon}\right)$ except that the 
volume constraint is [the original from $\left.\left(\mathcal{P}_{3}\right)\right] 1_{m}^{T} x \leq v$. (This problem clearly has optimal solutions for every $\varepsilon>0$ small enough.) Then, obviously,

$$
0 \leq c^{f, M}\left(x_{\varepsilon}^{*}, \tilde{s}_{\varepsilon}^{*}\right) \leq c^{f, M}\left(\hat{x}_{\varepsilon}^{*}, \hat{s}_{\varepsilon}^{*}\right)
$$

holds, and the sequence $\left\{c^{f, M}\left(\hat{x}_{\varepsilon}^{*}, \hat{s}_{\varepsilon}^{*}\right)\right\}$ is furthermore monotonically decreasing (hence upper bounded), since the feasible set of the corresponding problem increases monotonically with a decreasing value of $\varepsilon$.

Since $\left\{c^{f, M}\left(x_{\varepsilon}^{*}, \tilde{s}_{\varepsilon}^{*}\right)\right\}$ is bounded, so is the sequence $\left\{\left(\tilde{s}_{\varepsilon}^{*}, \tilde{\lambda}_{\varepsilon}^{*}\right)\right\}$. Further, by (37) and Theorem 3.1, if $\bar{x}$ is an arbitrary limit point of the sequence $\left\{x_{\varepsilon}^{*}\right\}$ then $\left\{\left(\tilde{s}_{\varepsilon}^{*}, \tilde{\lambda}_{\varepsilon}^{*}\right)\right\}$ converges to the optimal solution (cf. Theorem 2.1), say, $(\bar{s}, \bar{\lambda})$, to the problems $(\mathcal{C})_{(\bar{x})}\left(\omega_{\ell}\right), \ell \in \mathcal{L}$.

Consider next an arbitrary feasible solution $(x, \tilde{s}, \tilde{\lambda})$ to the problem $\left(\mathcal{P}_{3}\right)$, let $x_{\varepsilon}:=x+\varepsilon 1_{m}$, $\left(\tilde{s}_{\varepsilon}, \tilde{\lambda}_{\varepsilon}\right)$ be the optimal solution to the problems $(\mathcal{C})_{\left(x_{\varepsilon}\right)}\left(\omega_{\ell}\right), \ell \in \mathcal{L}$, and $\tilde{u}_{\varepsilon}$ the optimal solutions to the problems $(\mathcal{P})_{\left(x_{\varepsilon}\right)}\left(\omega_{\ell}\right), \ell \in \mathcal{L}$. Clearly, $\left(x_{\varepsilon}, \tilde{s}_{\varepsilon}, \tilde{\lambda}_{\varepsilon}\right)$ is a feasible solution to $\left(\mathcal{P}_{3}^{\varepsilon}\right)$. Corollary 3.2 implies that the sequence $\left\{\left(\tilde{s}_{\varepsilon}, \tilde{\lambda}_{\varepsilon}\right)\right\}$ of states converges to the limit state $(\tilde{s}, \tilde{\lambda})$. Further, Theorem 3.2 states that the sequence $\left\{\tilde{u}_{\varepsilon}\right\}$ converges to the least-energy displacement solution, here denoted $\tilde{u}$, to the problems $(\mathcal{P})_{(x)}\left(\omega_{\ell}\right), \ell \in \mathcal{L}$.

We then have that

$$
\begin{aligned}
c^{f, M}(\bar{x}, \bar{s}) & \leq \liminf _{\varepsilon \rightarrow 0} c^{f, M}\left(x_{\varepsilon}^{*}, \tilde{s}_{\varepsilon}^{*}\right) \\
& \leq \liminf _{\varepsilon \rightarrow 0} c^{f, M}\left(x_{\varepsilon}, \tilde{s}_{\varepsilon}\right) \\
& =\lim _{\varepsilon \rightarrow 0} c^{d, M}\left(x_{\varepsilon}, \tilde{u}_{\varepsilon}\right) \\
& =c^{d, M}(x, \tilde{u}) \\
& =c^{f, M}(x, \tilde{s}),
\end{aligned}
$$

where the inequalities follow from the lsc property of $c^{f, M}$ and the optimality of $\left(x_{\varepsilon}^{*}, \tilde{s}_{\varepsilon}^{*}, \tilde{\lambda}_{\varepsilon}^{*}\right)$ and feasibility of $\left(x_{\varepsilon}, \tilde{s}_{\varepsilon}, \tilde{\lambda}_{\varepsilon}\right)$ in the problem $\left(\mathcal{P}_{3}^{\varepsilon}\right)$, and the equalities follow from the identity between $c^{f, M}$ and $c^{d, M}$ on the equilibrium sets gr $\mathcal{S}_{\ell}, \ell \in \mathcal{L}$, the continuity of $c^{d, M}$, and again from the identity between $c^{f, M}$ and $c^{d, M}$ on the equilibrium set. By $(39),(\bar{x}, \bar{s})$ is optimal in $\left(\mathcal{P}_{3}\right)$. The convergence of the sequence $\left\{\left(x_{\varepsilon}^{*}, \tilde{s}_{\varepsilon}^{*}, \tilde{\lambda}_{\varepsilon}^{*}\right)\right\}$ to the optimal solution set of $\left(\mathcal{P}_{3}\right)$ then follows from its compactness. Since $c^{f, M}$ equals $c^{d, M}$ on gr $\mathcal{S}_{\ell}, \ell \in \mathcal{L}$, the last result follows also.

With the available proof techniques, an $\varepsilon$-perturbation of the volume constraint was needed in the definition of $\left(\mathcal{P}_{3}^{\varepsilon}\right)$, even though we suspect Theorem 5.2 may be true also without it. For instance, it can be shown that the $\varepsilon$-relaxation is not necessary with respect to the total volume constraint in the problem $\left(\mathcal{P}_{3}^{\varepsilon}\right)$ if $M=E^{-1}$, since, in this case, the design objective equals that of the problems $(\mathcal{C})_{(x)}\left(\omega_{\ell}\right), \ell \in \mathcal{L}$, whence we obtain that

$$
\begin{aligned}
c^{f}(\bar{x}, \bar{s}) & \leq \liminf _{\varepsilon \rightarrow 0} c^{f}\left(x_{\varepsilon}^{*}, \tilde{s}_{\varepsilon}^{*}\right) \\
& \leq \liminf _{\varepsilon \rightarrow 0} c^{f}\left(x_{\varepsilon}, \tilde{s}_{\varepsilon}\right) \\
& \leq \lim _{\varepsilon \rightarrow 0} c^{f}\left(x_{\varepsilon}, \tilde{s}\right) \\
& =c^{f}(x, \tilde{s}),
\end{aligned}
$$

where the equality follows from the continuity of $c^{f}(\cdot, \tilde{s})$ [cf. Lemma 3.1].

We finally note that in order to avoid checkerboard-like numerical instabilities, it may be necessary to slightly change the design parametrization by assigning the same thickness value to more than one finite element. For instance, triangular elements could be ordered in neighboring pairs and assigned the same thickness. 


\section{Concluding remarks and further research}

In this paper, we have considered quite general problems in structural topology optimization of unilaterally constrained mechanical structures. This class of optimization problems provides several difficulties, such as principal problems connected to the alteration of topology; for instance, many permissible designs lack equilibria. The remedy to this problem, achieved by providing and validating $\varepsilon$-perturbations that transform the problem statement to a sizing form, is one of the main themes of this paper. However, even if the topology has been fixed in the sizing problem, the design-to-state mappings are only locally Lipschitz continuous and generally not differentiable. This is due to the inclusion of unilateral constraints in the equilibrium formulations. Therefore, when including mechanical contact or cables, the nested versions of the structural optimization problems are not differentiable.

In the future we will investigate appropriate numerical solution algorithms for some of the problems investigated - a topic which is not covered in this paper. The monograph [LPR96] includes several algorithms for this class of problems, such as the implicit programming algorithm for the nested problem, and the penalty interior point algorithm for the simultaneous version of the problem, cf. also [OKZ98, HKP99a]. The nested problem can in principle be treated by any method from nondifferentiable optimization, for example, bundle methods ([OKZ98]) and subgradient methods ([CPW01]). Another possibility is to use smoothing (cf. [FJQ99, Hil00]), that is, to replace the equilibrium problem by a sequence of smooth approximations. Then any standard first-order algorithm, such as sequential explicit approximation methods, can be used for the nested problem.

As always in structural optimization, nonconvexity is a potential problem, in that first-order methods may terminate at non-global, local optima, corresponding to rather inefficient structures. Sometimes this seems to be especially pronounced in $\varepsilon$-relaxed topology optimization (cf. [StS01]) and in optimization in unilateral mechanics. In [Hil00], it was however reported that a smoothing modification may improve a first-order algorithm's ability to avoid (non-global) local optima that are due to the presence of unilateral constraints.

Compliance minimization is a rare special instance of the class of structural optimization problems, since it can be formulated as convex optimization problems and convex-concave saddle-point problems. Moreover, in the nested approach, the derivatives (when they exist) of compliance with respect to changes in designs, are very cheap to calculate. For other structural optimization problems, such as stress-constrained problems, the constraints are numerous, and the sensitivity analyses can be expected to require much more computational time.

In this paper, we handled stochastic forces through a straightforward discretization of the sample space. Other data can also be allowed to be random, such as the gaps $g_{1}$ or initial cable slacks $g_{2}$, as a way of accounting for mounting uncertainties. Moreover, alternative probability space discretizations, or other means to handle stochastic data, are possible and will be investigated, as well as the most natural choices of objective functions in the more general settings mentioned.

The aggregate effects on the proper choices of numerical procedures, of all the generalizations of design problems previously considered which we have either investigated in detail in this paper or only briefly mentioned, will be the subject of substantial forthcoming theoretical and numerical investigations. Some of the complications introduced because of the added complexity have already been mentioned in this section; some others, pertaining to the introduction of stochastic data, are discussed in [CPW01].

\section{Acknowledgments}

The authors would like to thank the anonymous referees for several valuable remarks. 


\section{References}

[Ach98] W. Achtziger, Multiple-load truss topology and sizing optimization: Some properties of minimax compliance, Journal of Optimization Theory and Applications, 98 (1998), pp. 255280.

[AuF90] J.-P. Aubin and H. Frankowska, Set-Valued Analysis, Birkhäuser, Boston, 1990.

[BGK+83] B. Bank, J. Guddat, D. Klatte, B. Kummer, And K. Tammer, Nonlinear parametric optimization, Birkhäuser Verlag, Basel, 1983.

[BSS93] M.S. BazaraA, H.D. Sherali, and C.M. Shetty, Nonlinear Programming: Theory and Algorithms, John Wiley \& Sons, New York, NY, 2nd edition, 1993.

[Bro66] F.E. BRowDER, Existence and approximation of solutions of nonlinear variational inequalities, Proceedings of the National Academy of Sciences, U.S.A., 56 (1966), pp. 1080-1086.

[Che95] G.D. Cheng, Some aspects of truss topology optimization, Structural Optimization, 10 (1995), pp. $173-179$.

[ChG97] G.D. Cheng And X. Guo, $\varepsilon$-relaxed approach in structural topology optimization, Structural Optimization, 13 (1997), pp. 258-266.

[CPW01] S. Christiansen, M. Patriksson, and L. Wynter, Stochastic bilevel programming in structural optimization, Structural and Multidisciplinary Optimization, 21 (2001), pp. 361-371.

[DGG64] W.S. Dorn, R.E. Gomory, And H.J. Greenberg, Automatic design of optimal structures, Journal de Mecanique, 3 (1964), pp. 25-52.

[DuB98] P. Duysinx And M.P. Bendsøe, Topology optimization of continuum structures with local stress constraints, International Journal for Numerical Methods in Engineering, 43 (1998), pp. 1453-1478.

[FJQ99] F. FACChIneI, H. JiAnG, AND L. QI, A smoothing method for mathematical programs with equilibrium constraints. Mathematical Programming, 85 (1999), pp. 107-134.

[FrW56] M. Frank And P. Wolfe, An algorithm for quadratic programming, Naval Research Logistics Quarterly, 3 (1956), pp. 95-110.

[Hil00] D. Hilding, A heuristic smoothing procedure for avoiding local optima in optimization of structures subject to unilateral constraints, Structural and Multidisciplinary Optimization, 20 (2000), pp. 29-36.

[HKP99a] D. Hilding, A. Klarbring, And J.-S. Pang, Minimization of maximum unilateral force, Computer Methods in Applied Mechanics and Engineering, 177 (1999), pp. 215-234.

[HKP99b] D. Hilding, A. Klarbring, and J. Petersson, Optimization of structures in unilateral contact, Applied Mechanics Reviews, 52 (1999), pp. 139-160.

[Kir90] U. KIRSCH, On singular topologies in optimum structural design, Structural Optimization, 2 (1990), pp. 133-142.

[LPR96] Z.-Q. Luo, J.-S. PAng, And D. RAlph, Mathematical Programs with Equilibrium Constraints, Cambridge University Press, Cambridge, UK, 1996.

[LuT92] Z.-Q. LuO And P. Tseng, Error bound and convergence analysis of matrix splitting algorithms for the affine variational inequality problem, SIAM Journal on Optimization, 2 (1992), pp. 4354

[LuT97] Z.-Q. Luo And P. Tseng, On a global projection-type error bound for the linear complementarity problem, Linear Algebra and Its Applications, 253 (1997), pp. 251-278.

[OKZ98] J. Outrata, M. Kočvara, and J. Zowe, Nonsmooth Approach to Optimization Problems with Equilibrium Constraints, Kluwer Academic Publishers, Dordrecht, 1998.

[PeH98] J. Petersson and J. Haslinger, An approximation theory for optimum sheets in unilateral contact, Quarterly of Applied Mathematics, 56 (2) (1998), pp. 309-325. 
[PeK94] J. Petersson And A. Klarbring, Saddle point to stiffness optimization of discrete structures including unilateral contact, Control and Cybernetics, 3 (1994), pp. 461-480.

[PeP97] J. Petersson and M. Patriksson, Topology optimization of sheets in contact by a subgradient method, International Journal of Numerical Methods in Engineering, 40 (1997), pp. 12951321.

[Rob80] S.M. Robinson, Strongly regular generalized equations, Mathematics of Operations Research, 5 (1980), pp. 43-62.

[Rob91] S.M. Robinson, An implicit-function theorem for a class of nonsmooth functions. Mathematics of Operations Research, 16 (1991), pp. 282-309.

[Roc70] R.T. Rockafellar, Convex Analysis, Princeton University Press, Princeton, NJ, 1970.

[Roz01] G.I.N. Rozvany, On design-dependent constraints and singular topologies, Structural and Multidisciplinary Optimization, 21 (2001), pp. 164-172.

[RoB94] G.I.N. Rozvany And T. BIRkER, On singular topologies in exact layout optimization, Structural Optimization, 8 (1994), pp. 228-235.

[Sta69] G. Stampacchia, Variational Inequalities, Pubblicazioni IAC, Serie III - N. 25, Rome, 1969.

[StS01] M. Stolpe and K. Svanberg, On the trajectories of the $\varepsilon$-relaxation approach for stressconstrained truss topology optimization, Structural and Multidisciplinary Optimization, 21 (2001), pp. 140-151.

[Sva94] K. Svanberg, Global convergence of the stress ratio method for truss sizing, Structural Optimization, 8 (1994), pp. 60-68.

[SvG68] G. Sved And Z. Ginos, Structural optimization under multiple loading, International Journal of Mechanical Sciences, 10 (1968), pp. 803-805. 